\title{
MOLECULAR LINE OBSERVATIONS OF INFRARED DARK CLOUDS. II. PHYSICAL CONDITIONS
}

\author{
David Gibson ${ }^{1}$, René Plume ${ }^{1,3}$, Edwin Bergin ${ }^{2}$, Sarah Ragan $^{2}$, and Natalie Evans ${ }^{1}$ \\ ${ }^{1}$ Department of Physics and Astronomy, University of Calgary, 2500 University Drive NW, Calgary, Alberta T2N 1N4, Canada \\ ${ }^{2}$ Department of Astronomy, University of Michigan, 500 Church Street, Ann Arbor, MI 48109, USA \\ ${ }^{3}$ Max Planck Institute for Astronomy, Köningstuhl 17, 69117 Heidelberg, Germany \\ Received 2009 June 26; accepted 2009 August 24; published 2009 October 8
}

\begin{abstract}
Using a source selection biased toward high-mass star-forming regions, we used a large velocity gradient code to calculate the $\mathrm{H}_{2}$ densities and CS column densities for a sample of Midcourse Space Experiment $8 \mu \mathrm{m}$ infrared dark cores. Our average $\mathrm{H}_{2}$ density and CS column density were $1.14 \times 10^{6} \mathrm{~cm}^{-3}$ and $1.21 \times 10^{13} \mathrm{~cm}^{-2}$, respectively. In addition, we have calculated the Jeans mass and Virial mass for each core to get a better understanding of their gravitational stability. We found that core masses calculated from observations of $\mathrm{N}_{2} \mathrm{H}^{+} J=1 \rightarrow 0$ and $\mathrm{C}^{18} \mathrm{O} J=1 \rightarrow 0$ by Ragan et al. (Paper I) were sufficient for collapse, though most regions are likely to form protoclusters. We have explored the star-forming properties of the molecular gas within our sample and find some diversity which extends the range of infrared dark clouds from the very massive clouds that will create large clusters, to clouds that are similar to some of our local counterparts (e.g., Serpens, Ophiuchus).
\end{abstract}

Key words: ISM: clouds - ISM: molecules - stars: formation

Online-only material: color figure

\section{INTRODUCTION}

Over the past decade, infrared dark clouds (IRDCs) have become to be recognized as the sites of clustered star formation in our galaxy (Rathborne et al. 2006; Ragan et al. 2009; Butler \& Tan 2009). IRDCs are important not only in understanding the earliest stage of the birth of stellar clusters, which is the dominant mode of galactic star formation (Lada \& Lada 2003), but also as the primary sites of massive star formation. Massive star formation plays an integral role in the evolution of galaxies as they are responsible for energizing the interstellar medium (ISM; McKee 1986), producing the heavy elements (Dopita 1991), and have been suggested to regulate the rate of star formation (McKee \& Tan 2003). Despite their importance, a variety of observational and theoretical issues have made massive star formation a difficult topic to address. In particular, the greater distances toward massive star-forming regions, faster evolutionary timescales, and source confusion (e.g., Garay \& Lizano 1999) make it difficult to isolate the earliest stages of massive star formation. Since IRDCs represent the earliest stages of star cluster formation they provide fertile ground for understanding the beginnings of high-and low-mass stellar birth (Menten et al. 2005, Bergin \& Tafalla 2007).

There have been several studies of IRDCs that have characterized the masses and column density structure in these objects with the ultimate aim of understanding clustered and massive star formation (Sridharan et al. 2005; Pillai et al. 2006; Rathborne et al. 2006; Ragan et al. 2006; Du \& Yang 2008). What is clear is that these objects are massive, with masses in excess of $100 M_{\odot}$ which is comparable to the envelopes around some massive protostars. The velocity line widths of $1-3 \mathrm{~km} \mathrm{~s}^{-1}$ are also characteristically below that of molecular gas surrounding massive stars, which is believed to be related to the fact that the IR dark clouds are in an earlier phase of star formation. In addition, they are often associated with the signposts of star formation including masers (Beuther et al. 2002; Wang et al. 2006), outflows (Beuther \& Sridharan 2007), and embedded infrared point sources (Beuther \& Steinacker 2007, Chambers et al. 2009; Ragan et al. 2009).
Since IRDCs have only been recently recognized there is a basic lack of large-scale information on various physical characteristics, such as the temperature and densities, that can be reliably determined via well-known molecular-line techniques. Pillai et al. (2006, 2007) and Sridharan et al. (2002) used the $\mathrm{NH}_{3}$ inversion transitions to explore temperature variations within a sample of 32 and 40 IRDCs respectively and found that gas temperatures are typically $20 \mathrm{~K}$. Direct determination of the densities from $\mathrm{H}_{2} \mathrm{CO}$ (Carey et al. 1998), $\mathrm{CH}_{3} \mathrm{OH}$ (Leurini et al. 2007), and CS (Rathborne et al. 2008) are available for about $10-20$ objects with densities found to be in excess of $10^{5} \mathrm{~cm}^{-3}$. It is the aim of this work to provide some of this basic information to further illuminate the general characteristics of IRDCs in the context of massive star formation. In particular, we will use multi-line observations of CS to estimate the gas density for a larger sample of 41 infrared dark clouds isolated from the MSX database by Ragan et al. (2006). Using this information, we will explore the diversity amongst a modest portion of the IRDC population and grossly characterize the stability of these objects against gravitational collapse.

In Sections 2 and 3, we will present our observations and place the sources into different categories of molecular emission morphologies based on observations from Ragan et al. (2006; hereafter Paper I) and new CS observations. We will then present the results of large velocity gradient (LVG) analysis of these cores to constrain their $\mathrm{H}_{2}$ densities and CS column densities. In Section 4, we will present the evidence that confirms several of our selected cores are actually massive star-forming regions, and assign relative chemical ages to these cores using a chemical evolution model.

\section{OBSERVATIONS}

\subsection{Source Selection}

There have been numerous studies of the initial conditions of high-mass star formation using a variety of criteria with which to select sources. For example, some have used Infrared Astronomical Satellite (IRAS) colors and/or water masers as signposts of star formation (e.g., Molinari et al. 1996, Plume 
et al. 1992, 1997; Knez et al. 2002; Mueller et al. 2002; Sridharan et al. 2002). Others have surveyed a sample of infrared dark cores (e.g., Redman et al. 2002; Sridharan et al. 2005; Rathborne et al. 2006; Pillai et al. 2006) and some have selected their sample from the mapping of molecular cores (e.g., Wu et al. 2000; Garay et al. 2004). All of these studies, however, have both benefits and drawbacks. For example, the water maser studies could not disentangle massive young stellar objects (YSOs) that have already formed from those in the initial stages of collapse. On the other hand, the IRDC studies cannot confirm that the clouds mapped will indeed form massive YSOs.

In Paper I, we presented a biased sample of potential massive protostellar cores from the $8.8 \mu \mathrm{m}$ images of the Midcourse Space Experiment (MSX; Price et al. 1996) satellite. This sample is biased in that, to maximize the possibility that our sample actually contains massive prestellar objects, we have constrained our search to IR dark cores that are located close to Ultra Compact HII (UCHII) regions on the plane of the sky. Given that massive stars tend to form in clusters (Lada et al. 1993), current massive star-forming regions are excellent places to look for potential massive protostellar cores. In particular, we looked for cores that were IR dark at $8 \mu \mathrm{m}$ (suggesting either starless cores or deeply embedded YSOs) that were near UCHII regions (signposts of very young massive stars; Churchwell 2002).

Using this criterion, we identified 114 objects of which only a small fraction ( $15 \%$ ) have known associations with radio sources or masers. Of these, 41 were selected due to their compactness $\left(R_{\text {core }}<2.1 \mathrm{pc}\right)$, and relatively high opacity ( $\tau \gtrsim 0.4$ at $\left.8.8 \mu \mathrm{m}\right)$. In this paper, we present new observations of the 41 IRDCs presented in Paper I.

\subsection{Molecular Line Observations}

In Paper I, we presented observations of $\mathrm{C}^{18} \mathrm{O} J=1 \rightarrow$ $0(v=109.782 \mathrm{GHz}), \mathrm{CS} J=2 \rightarrow 1(v=97.981 \mathrm{GHz})$ and $\mathrm{N}_{2} \mathrm{H}^{+} J=1 \rightarrow 0(v=93.173 \mathrm{GHz})$ which were observed at the $14 \mathrm{~m}$ FCRAO in 2002 February, 2002 May, and 2002 December. Using the 16 element focal plane array receiver Second Quabbin Optical (or Observatory) Imaging Array (SEQUOIA), each region was mapped with $17 \times 17$ point maps spanning $400^{\prime \prime} \times 400^{\prime \prime}$, typically to a $1 \sigma \mathrm{rms}$ noise level of $\sim 0.05-0.1 \mathrm{~K}$. We used the narrowband correlator backend, which was configured to a velocity resolution of $\sim 0.13 \mathrm{~km} \mathrm{~s}^{-1}$. System temperatures were typically 200-300 K. Main beam efficiencies $\left(\eta_{\mathrm{mb}}\right)$ were assumed to be 0.48 , taken from standard FCRAO values. The FCRAO beamsize at these frequencies is $\Theta_{\mathrm{FWHM}} \sim$ $45^{\prime \prime}$. The emission from CO $J=1 \rightarrow 0(v=115.271 \mathrm{GHz})$ was also observed at the $14 \mathrm{~m}$ FCRAO over the same time period as the other observations using the same configurations, except the ${ }^{12} \mathrm{CO}$ observations were performed in poorer weather conditions $\left(T_{\text {sys }} \sim 750 \mathrm{~K}\right)$. Though not used in Paper I, these $\mathrm{CO}$ observations have been used in this publication (see Table 1 for results).

In this paper, we focus on new $\mathrm{CS}$ observations. Emission from CS $J=3 \rightarrow 2(v=146.969 \mathrm{GHz})$ and $\mathrm{CS} J=5 \rightarrow 4$ $(v=244.936 \mathrm{GHz})$ was observed at the central position of the $M S X$ cores (constrained using the peak absorption in the $M S X$ band $A$ image) in 2002 October, 2003 February, 2003 March, 2003 June, and 2004 April. However, in a few sources the MSX core was not co-located with the intensity peak of the previously obtained $\mathrm{CS}, \mathrm{CO}$, and $\mathrm{C}^{18} \mathrm{O}$ molecular line maps. In these cases, the new CS observations were taken on the center of the $M S X$ core, the position of which is listed as an offset $(\Delta \alpha, \Delta \delta)$ from the center of the previously obtained molecular line maps.
The CS $J=3 \rightarrow 2$ was observed at the Kitt Peak $12 \mathrm{~m}$ telescope using filter bank spectrometers FB12 and FB22, as well as the Millimeter Auto Correlator (MAC). These instruments were configured to a velocity resolution of $\sim 0.2 \mathrm{~km} \mathrm{~s}^{-1}$, $0.51 \mathrm{~km} \mathrm{~s}^{-1}$, and $0.05 \mathrm{~km} \mathrm{~s}^{-1}$, respectively, and typically yield a $1 \sigma \mathrm{rms}$ noise level of $\sim 0.05-0.1 \mathrm{~K}$. System temperatures were typically $300-400 \mathrm{~K}$, with a beamsize of $\Theta_{\mathrm{FWHM}}=45^{\prime \prime}$ and a main beam efficiency of $\eta_{\mathrm{mb}}=0.48$. CS $J=5 \rightarrow 4$ was observed at the $10 \mathrm{~m}$ Heinrich Hertz Submillimeter Telescope (HHSMT) using acousto-optical spectrometers B and C, and the Chirp Transform Spectrometer, configured to a velocity resolution of $0.5876 \mathrm{~km} \mathrm{~s}^{-1}, 0.1481 \mathrm{~km} \mathrm{~s}^{-1}$, and $0.057 \mathrm{~km} \mathrm{~s}^{-1}$, respectively. The central position of each $M S X$ core was observed with a $37^{\prime \prime}$ beam $\left(\eta_{\mathrm{MB}}=0.82\right)$, with a typical rms noise level of $\sim 0.05-0.5 \mathrm{~K}$. System temperatures were typically 300 $400 \mathrm{~K}$, however the observations made in 2003 June were performed in poorer weather conditions raising the temperature $\left(T_{\text {sys }} \sim 800 \mathrm{~K}\right)$. Results of these observations can be found in Tables 2 and 3 .

The emission from CS $J=7 \rightarrow 6(v=342.882 \mathrm{GHz})$ was observed at the $10.4 \mathrm{~m}$ Caltech Submillimeter Observatory (CSO) in 2002 July, using the $280-400 \mathrm{GHz}$ receiver and the acousto-optical spectrometer (AOS) backend configured to a velocity resolution of $\sim 0.042 \mathrm{~km} \mathrm{~s}^{-1}$. The central position of each $M S X$ core was observed with a $24.6^{\prime \prime}$ beam $\left(\eta_{\mathrm{MB}}=0.75\right)$. Typical system temperatures were $600-650 \mathrm{~K}$. Results of these observations can be found in Table 3 .

All data are presented in units of $T_{A}{ }^{*}$ (Kutner \& Ulich 1981). However, to compare our observations to model predictions and to calculate physical conditions, we must include the main beam coupling efficiency $\left(\eta_{\mathrm{mb}}\right)$ such that $T_{\mathrm{mb}}=T_{A}^{*} / \eta_{\mathrm{mb}}$. All data were calibrated with the standard chopper wheel method.

\section{RESULTS}

\subsection{Molecular Emission}

Tables 2 and 3 show the results of Gaussian fits to the observed CS transitions. The tables consist of the following entries (labeled as Columns 1-11): (1) core name, (2) and (3) the position offsets ( $\Delta \alpha$ and $\Delta \delta$, respectively), (4) and (8) the antenna temperature $\left(T_{a}^{*}\right),(5)$ and (9) the full width at halfmaximum (FWHM), (6) and (10) the source $\mathrm{v}_{\mathrm{lsr}}$, and (7) and (11) the integrated intensity $\left(\int T_{a}^{*} d v\right)$. Average line widths at the core's central position are $2.85 \mathrm{~km} \mathrm{~s}^{-1}$ for CS $J=2 \rightarrow 1$, $2.68 \mathrm{~km} \mathrm{~s}^{-1}$ for CS $J=3 \rightarrow 2,3.09 \mathrm{~km} \mathrm{~s}^{-1}$ for CS $J=5 \rightarrow 4$, and $2.93 \mathrm{~km} \mathrm{~s}^{-1}$ for CS $J=7 \rightarrow 6$.

In Paper I, we presented a catalog of maps displaying the spatial distributions of $\mathrm{C}^{18} \mathrm{O} J=1 \rightarrow 0, \mathrm{CS} J=2 \rightarrow 1$, and $\mathrm{N}_{2} \mathrm{H}^{+} J=1 \rightarrow 0$ for each source. Based on the spatial distributions, we are able to separate our sources into four broad categories. $\mathrm{CO}$ is not included in this categorization process since it is not as useful a discriminant as is $\mathrm{C}^{18} \mathrm{O}$. Table 4 shows each core's morphological category based upon the following criteria:

1. Category 1. CS $J=2 \rightarrow 1$ and/or $\mathrm{C}^{18} \mathrm{O} J=1 \rightarrow 0$ emission detectable and centralized on the $M S X$ dark core, but no detectable $\mathrm{N}_{2} \mathrm{H}^{+} J=1 \rightarrow 0$. Five cores fall in this category: G06.26-0.51, G09.16+0.06, G14.33-0.57, $\mathrm{G} 24.16+0.08$, and G37.44+0.14.

2. Category 2. Centralized $\mathrm{CS} J=2 \rightarrow 1$ and/or $\mathrm{C}^{18} \mathrm{O}$ $J=1 \rightarrow 0$ emission, with detectable $\mathrm{N}_{2} \mathrm{H}^{+} J=1$ $\rightarrow 0$ emission. Seventeen cores fall in this category: G09.21-0.22, G09.28-0.15, G09.86-0.04, G10.99-0.09, 
Table 1

Observational Parameters at Center of $M S X$ Core for CO $J=1 \rightarrow 0$

\begin{tabular}{|c|c|c|c|c|c|c|}
\hline $\begin{array}{l}\text { Core } \\
\text { (1) }\end{array}$ & $\begin{array}{l}\Delta \alpha \\
\left({ }^{\prime \prime}\right) \\
(2)\end{array}$ & $\begin{array}{l}\Delta \delta \\
\left({ }^{\prime \prime}\right) \\
(3)\end{array}$ & $\begin{array}{l}T_{a}^{*} \\
(\mathrm{~K}) \\
(4)\end{array}$ & $\begin{array}{c}\Delta v \\
\left(\mathrm{~km} \mathrm{~s}^{-1}\right) \\
(5)\end{array}$ & $\begin{array}{c}v_{\mathrm{lsr}} \\
\left(\mathrm{km} \mathrm{s}^{-1}\right) \\
(6)\end{array}$ & $\begin{array}{c}\int T_{a}^{*} d v \\
\left(\mathrm{~K} \mathrm{~km} \mathrm{~s}^{-1}\right) \\
(7)\end{array}$ \\
\hline G05.85-0.23 & 25 & -25 & 2.25 & 3.25 & 16.85 & 7.76 \\
\hline G06.26-0.51 & 0 & 0 & 3.55 & 4.99 & 21.89 & 18.85 \\
\hline G09.16+0.06 & 0 & 0 & 2.98 & 3.14 & 31.89 & 9.96 \\
\hline G09.21-0.22 & 0 & 0 & $\ldots$ & $\ldots$ & $\ldots$ & $\ldots$ \\
\hline G09.28-0.15 & 0 & 0 & $\ldots$ & $\ldots$ & $\ldots$ & $\ldots$ \\
\hline G09.86-0.04 & 0 & 0 & $\ldots$ & $\ldots$ & $\ldots$ & $\ldots$ \\
\hline G09.88-0.11 & 0 & 0 & $\ldots$ & $\ldots$ & $\ldots$ & $\ldots$ \\
\hline G10.59-0.31 & 0 & 0 & $\ldots$ & $\ldots$ & $\ldots$ & $\ldots$ \\
\hline G10.70-0.33 & 0 & 0 & $\ldots$ & $\ldots$ & $\ldots$ & $\ldots$ \\
\hline G10.99-0.09 & 0 & 0 & 1.11 & 4.28 & 29.20 & 4.60 \\
\hline $\mathrm{G} 12.22+0.14$ & 0 & 0 & $\ldots$ & $\ldots$ & $\ldots$ & $\ldots$ \\
\hline G12.50-0.22 & 0 & -25 & 0.88 & 2.51 & 35.82 & 3.65 \\
\hline G14.33-0.57 & 0 & 0 & 4.60 & 8.09 & 21.27 & 39.61 \\
\hline G19.28-0.39 & 0 & 0 & $\ldots$ & $\ldots$ & $\ldots$ & $\ldots$ \\
\hline G19.37-0.03 & 0 & 0 & $\ldots$ & $\ldots$ & $\ldots$ & $\ldots$ \\
\hline G19.40-0.01 & 0 & 0 & $\ldots$ & $\ldots$ & $\ldots$ & $\ldots$ \\
\hline G23.37-0.29 & 0 & 0 & 2.69 & 7.16 & 76.57 & 20.47 \\
\hline G23.48-0.53 & 0 & 0 & $\ldots$ & $\ldots$ & $\ldots$ & $\ldots$ \\
\hline $\mathrm{G} 24.05-0.22$ & 25 & 0 & 3.32 & 5.84 & 81.52 & 20.61 \\
\hline $\mathrm{G} 24.16+0.08$ & 0 & 0 & 2.10 & 9.64 & 109.55 & 21.56 \\
\hline G25.99-0.06 & 25 & 25 & $\ldots$ & $\ldots$ & $\ldots$ & $\ldots$ \\
\hline G30.14-0.07 & 0 & 0 & $\ldots$ & $\ldots$ & $\ldots$ & $\ldots$ \\
\hline G30.49-0.39 & 0 & 0 & $\ldots$ & $\ldots$ & $\ldots$ & $\ldots$ \\
\hline G30.53-0.27 & 0 & 0 & $\ldots$ & $\ldots$ & $\ldots$ & $\ldots$ \\
\hline G30.89+0.14 & 0 & 0 & $\ldots$ & $\ldots$ & $\ldots$ & $\ldots$ \\
\hline G30.98-0.15 & -25 & 0 & $\ldots$ & $\ldots$ & $\ldots$ & $\ldots$ \\
\hline G31.02-0.12 & -25 & 0 & 3.52 & 4.03 & 75.98 & 15.09 \\
\hline G32.01+0.05 & 0 & 0 & 7.10 & 7.78 & 96.25 & 58.80 \\
\hline G33.82-0.22 & 0 & 0 & $<0.46$ & $\ldots$ & $\ldots$ & $\ldots$ \\
\hline G34.63-1.03 & 0 & 0 & 1.19 & 2.35 & 11.58 & 2.97 \\
\hline G34.74-0.12 & -25 & 0 & 2.25 & 9.78 & 77.35 & 23.43 \\
\hline G34.78-0.80 & 0 & 0 & 3.43 & 4.56 & 43.30 & 42.32 \\
\hline G35.20-0.72 & 0 & 0 & 3.14 & 6.28 & 32.86 & 21.00 \\
\hline G37.44+0.14 & 0 & 0 & 7.67 & 2.51 & 39.96 & 20.46 \\
\hline G37.89-0.15 & 0 & -25 & 2.18 & 1.25 & 13.08 & 2.91 \\
\hline G43.64-0.82 & 0 & 0 & $\ldots$ & $\ldots$ & $\ldots$ & $\ldots$ \\
\hline G43.78+0.05 & 0 & 0 & $\ldots$ & $\ldots$ & $\ldots$ & $\ldots$ \\
\hline G50.07+0.06 & 0 & 0 & $\ldots$ & $\ldots$ & $\ldots$ & $\ldots$ \\
\hline G53.88-0.18 & 0 & 0 & $\ldots$ & $\ldots$ & $\ldots$ & $\ldots$ \\
\hline G75.75+0.75 & 0 & 0 & $\ldots$ & $\ldots$ & $\ldots$ & $\ldots$ \\
\hline G76.38+0.63 & 0 & 0 & $\ldots$ & $\ldots$ & $\ldots$ & $\ldots$ \\
\hline
\end{tabular}

$\mathrm{G} 12.22+0.14, \mathrm{G} 12.50-0.22, \mathrm{G} 19.37-0.03, \mathrm{G} 23.37-0.29$, G24.05-0.22, G25.99-0.06, G30.98-0.15, G32.01+0.05, G34.63-1.03, G34.74-0.12, G34.78-0.80, G35.20-0.72, and G37.89-0.15.

3. Category 3. Strong $\mathrm{N}_{2} \mathrm{H}^{+} J=1 \rightarrow 0$ emission, with reduced $\mathrm{CS} J=2 \rightarrow 1$ and $\mathrm{C}^{18} \mathrm{O} J=1 \rightarrow 0$ abundances based on their average column densities (see Table 4 for complete list of column densities). Five cores fall in this category: G05.85-0.23, G19.40-0.01, G23.48-0.53, G30.89+0.14, and G31.02-0.12.

4. Category 4. Little to no emission was detected in any of the molecular transitions observed. Fourteen cores fall in this category: G09.88-0.11, G10.59-0.31, G10.70-0.33, G19.28-0.39, G30.14-0.07, G30.49-0.39, G30.53-0.27, G33.82-0.22, G43.64-0.82, G43.78+0.05, G50.07+0.06, G53.88-0.18, G75.75+0.75, and G76.38+0.63. These cores are not included in Table 4.

In several cases, there were examples of multiple velocity components (e.g., G31.02-0.12, see Figure 1) likely due to line-of-sight clouds. In these cases, the velocity of each core was determined by analyzing the emission from multiple molecular lines for coincidence. We have made the assumption that CS, as a tracer of dense gas, preferentially selects the presumably dense core rather than the low-density line-of-sight emission. Figure 1 shows an example of this analysis.

In some other cases, molecular line emission peaks off the central position of the core (G10.59-0.31, 35.20-0.72, for example). This behavior could be caused by either a nearby object heating the surface of the cloud, internal heating from an embedded protostar, or abundance gradients causing the molecular emission to peak off the cold core center. The existence of possible embedded protostars has been explored in a sub-sample of these objects by Ragan et al. (2009). However in these cases, we still believe that the molecular emission is associated with the MSX cores and have been categorized accordingly. Alternatively, from the morphologies of G19.40-0.01 in Figure 1 from Paper I, for example, it is quite obvious that although the IR dark core is located in the center of the image, the molecular emission is centered on a 
Table 2

Observational Parameters at Center of $M S X$ Core for CS $J=2 \rightarrow 1$ and CS $J=3 \rightarrow 2$

\begin{tabular}{|c|c|c|c|c|c|c|c|c|c|c|}
\hline \multirow[b]{2}{*}{ Core } & \multirow[b]{2}{*}{$\begin{array}{l}\Delta \alpha \\
\left({ }^{\prime \prime}\right)\end{array}$} & \multirow[b]{2}{*}{$\begin{array}{l}\Delta \delta \\
\left({ }^{\prime \prime}\right)\end{array}$} & \multicolumn{4}{|c|}{$\mathrm{CS} J=2 \rightarrow 1$} & \multicolumn{4}{|c|}{$\mathrm{CS} J=3 \rightarrow 2$} \\
\hline & & & $\begin{array}{l}T_{a}^{*} \\
(\mathrm{~K}) \\
(4) \\
\end{array}$ & $\begin{array}{c}\Delta v \\
\left(\mathrm{~km} \mathrm{~s}^{-1}\right) \\
(5)\end{array}$ & $\begin{array}{c}v_{\mathrm{lsr}} \\
\left(\mathrm{km} \mathrm{s}^{-1}\right) \\
(6)\end{array}$ & $\begin{array}{c}\int T_{a}^{*} d v \\
\left(\mathrm{~K} \mathrm{~km} \mathrm{~s}{ }^{-1}\right) \\
(7)\end{array}$ & $\begin{array}{l}T_{a}^{*} \\
(\mathrm{~K}) \\
(8) \\
\end{array}$ & $\begin{array}{c}\Delta v \\
\left(\mathrm{~km} \mathrm{~s}^{-1}\right) \\
(9)\end{array}$ & $\begin{array}{c}v_{\mathrm{lsr}} \\
\left(\mathrm{km} \mathrm{s}^{-1}\right) \\
(10)\end{array}$ & $\begin{array}{c}\int T_{a}^{*} d v \\
\left(\mathrm{~K} \mathrm{~km} \mathrm{~s}^{-1}\right) \\
(11)\end{array}$ \\
\hline G05.85-0.23 & 25 & -25 & 0.21 & 2.20 & 16.90 & 0.47 & $\ldots$ & $\ldots$ & $\ldots$ & $\ldots$ \\
\hline G06.26-0.51 & 0 & 0 & 0.37 & 4.28 & 23.13 & 1.70 & 0.45 & 4.33 & 22.43 & 2.06 \\
\hline G09.16+0.06 & 0 & 0 & 0.33 & 1.13 & 31.18 & 0.40 & 0.32 & 1.76 & 31.08 & 0.59 \\
\hline G09.21-0.22 & 0 & 0 & 0.42 & 2.70 & 42.70 & 1.21 & 0.58 & 2.43 & 43.00 & 1.50 \\
\hline G09.28-0.15 & 0 & 0 & 0.47 & 2.70 & 41.33 & 1.36 & 0.36 & 2.72 & 41.59 & 1.03 \\
\hline G09.86-0.04 & 0 & 0 & 0.62 & 2.37 & 17.80 & 1.56 & 0.73 & 2.21 & 17.83 & 1.73 \\
\hline G09.88-0.11 & 0 & 0 & 0.30 & 1.80 & 17.30 & 0.54 & $\ldots$ & $\ldots$ & $\ldots$ & $\ldots$ \\
\hline G10.59-0.31 & 0 & 0 & $<0.20$ & $\ldots$ & $\ldots$ & $\ldots$ & $\ldots$ & $\ldots$ & $\ldots$ & $\ldots$ \\
\hline G10.70-0.33 & 0 & 0 & $<0.11$ & $\ldots$ & $\ldots$ & $\ldots$ & $\ldots$ & $\ldots$ & $\ldots$ & $\ldots$ \\
\hline G10.99-0.09 & 0 & 0 & 0.22 & 4.15 & 29.22 & 0.99 & 0.34 & 2.68 & 29.70 & 0.97 \\
\hline G12.22+0.14 & 0 & 0 & 0.75 & 2.26 & 39.66 & 1.80 & 0.96 & 2.17 & 40.04 & 2.21 \\
\hline G12.50-0.22 & 0 & -25 & 0.44 & 2.64 & 35.97 & 1.23 & 0.48 & 1.95 & 35.99 & 0.99 \\
\hline G14.33-0.57 & 0 & 0 & 0.51 & 2.34 & 19.58 & 1.26 & $\ldots$ & $\ldots$ & $\ldots$ & $\ldots$ \\
\hline G19.28-0.39 & 0 & 0 & 0.24 & 1.10 & 54.00 & 0.26 & 0.10 & 1.04 & 53.24 & 0.22 \\
\hline G19.37-0.03 & 0 & 0 & 0.73 & 3.63 & 26.97 & 2.82 & 1.36 & 4.20 & 26.82 & 6.05 \\
\hline G19.40-0.01 & 0 & 0 & 0.27 & 3.05 & 26.49 & 0.87 & $\ldots$ & $\ldots$ & $\ldots$ & $\ldots$ \\
\hline G23.37-0.29 & 0 & 0 & 0.44 & 4.67 & 77.88 & 2.20 & $\ldots$ & $\ldots$ & $\ldots$ & $\ldots$ \\
\hline G23.48-0.53 & 0 & 0 & 0.17 & 4.18 & 63.78 & 0.23 & $<0.16$ & $\ldots$ & $\ldots$ & $\ldots$ \\
\hline G24.05-0.22 & 25 & 0 & 0.26 & 2.70 & 81.67 & 0.75 & 0.40 & 2.74 & 81.57 & 1.17 \\
\hline G24.16+0.08 & 0 & 0 & $<0.10$ & $\ldots$ & $\ldots$ & $\ldots$ & $<0.09$ & $\ldots$ & $\ldots$ & $\ldots$ \\
\hline G25.99-0.06 & 25 & 25 & 0.35 & 2.20 & 90.18 & 0.82 & 0.41 & 2.85 & 89.78 & 1.26 \\
\hline G30.14-0.07 & 0 & 0 & $<0.11$ & $\ldots$ & $\ldots$ & $\ldots$ & $\ldots$ & $\ldots$ & $\ldots$ & $\ldots$ \\
\hline G30.49-0.39 & 0 & 0 & $<0.10$ & $\ldots$ & $\ldots$ & $\ldots$ & $<0.07$ & $\ldots$ & $\ldots$ & $\ldots$ \\
\hline G30.53-0.27 & 0 & 0 & 0.24 & 7.30 & 102.90 & 1.73 & $\ldots$ & $\ldots$ & $\ldots$ & $\ldots$ \\
\hline G30.89+0.14 & 0 & 0 & 0.15 & 0.74 & 95.76 & 0.39 & $\ldots$ & $\ldots$ & $\ldots$ & $\ldots$ \\
\hline G30.98-0.15 & -25 & 0 & 0.46 & 3.58 & 77.77 & 1.74 & 0.46 & 3.10 & 77.33 & 1.50 \\
\hline G31.02-0.12 & -25 & 0 & 0.21 & 3.29 & 76.73 & 0.72 & 0.16 & 3.30 & 77.16 & 0.78 \\
\hline G32.01+0.05 & 0 & 0 & 0.50 & 5.55 & 95.83 & 2.94 & 0.47 & 5.37 & 95.49 & 2.68 \\
\hline G33.82-0.22 & 0 & 0 & 0.45 & 0.95 & 11.51 & 0.45 & $\ldots$ & $\ldots$ & $\ldots$ & $\ldots$ \\
\hline G34.63-1.03 & 0 & 0 & $<0.09$ & $\ldots$ & $\ldots$ & $\ldots$ & $<0.08$ & $\ldots$ & $\ldots$ & $\ldots$ \\
\hline G34.74-0.12 & -25 & 0 & 0.28 & 3.78 & 78.67 & 1.13 & 0.24 & 3.16 & 79.23 & 0.80 \\
\hline G34.78-0.80 & 0 & 0 & 0.43 & 3.58 & 43.43 & 1.64 & 0.65 & 3.02 & 43.35 & 2.08 \\
\hline G35.20-0.72 & 0 & 0 & 0.52 & 3.26 & 33.21 & 1.82 & $\ldots$ & $\ldots$ & $\ldots$ & $\ldots$ \\
\hline G37.44+0.14 & 0 & 0 & 0.40 & 1.12 & 40.03 & 0.48 & 0.29 & 1.82 & 40.11 & 0.55 \\
\hline G37.89-0.15 & 0 & -25 & 0.57 & 0.66 & 12.96 & 0.40 & 0.31 & 1.00 & 12.95 & 0.32 \\
\hline G43.64-0.82 & 0 & 0 & $<0.10$ & $\ldots$ & $\ldots$ & $\ldots$ & $<0.06$ & $\ldots$ & $\ldots$ & $\ldots$ \\
\hline G43.78+0.05 & 0 & 0 & $<0.10$ & $\ldots$ & $\ldots$ & $\ldots$ & $<0.05$ & $\ldots$ & $\ldots$ & $\ldots$ \\
\hline G50.07+0.06 & 0 & 0 & $<0.12$ & $\ldots$ & $\ldots$ & $\ldots$ & 0.54 & 1.63 & 54.66 & 0.05 \\
\hline G53.88-0.18 & 0 & 0 & $<0.13$ & $\ldots$ & $\ldots$ & $\ldots$ & $<0.08$ & $\ldots$ & $\ldots$ & $\ldots$ \\
\hline G75.75+0.75 & 0 & 0 & $<0.08$ & $\ldots$ & $\ldots$ & $\ldots$ & $<0.05$ & $\ldots$ & $\ldots$ & $\ldots$ \\
\hline G76.38+0.63 & 0 & 0 & $<0.09$ & $\ldots$ & $\ldots$ & $\ldots$ & $<0.07$ & $\ldots$ & $\ldots$ & $\ldots$ \\
\hline
\end{tabular}

secondary source below it. Other examples of this can be found in G9.21-0.22 and G32.01+0.05. However, in these cases, despite the molecular emission appearing to be centered on a secondary source, they have been classified as category 2 objects as they still exhibit strong emission of all tracer molecules on the core center.

\subsection{Physical Conditions}

In Paper I, we presented simple local thermodynamic equilibrium (LTE) calculations of the $\mathrm{C}^{18} \mathrm{O}, \mathrm{N}_{2} \mathrm{H}^{+}$, and CS column densities and used these to extract the average $\mathrm{H}_{2}$ column density and cloud mass. Table 4, Columns 3 and 4 reproduces the results of the $\mathrm{C}^{18} \mathrm{O}$ and $\mathrm{N}_{2} \mathrm{H}^{+}$column density calculations, with $\mathrm{C}^{18} \mathrm{O}$ converted to $\mathrm{CO}$ assuming an abundance ratio of $N_{\mathrm{CO}}=$ $500 N_{\mathrm{C}^{18} \mathrm{O}}$. Here we present a more detailed analysis of the emission from multiple CS transitions to more accurately determine the gas density. We have presented only the 27 cores that fall in categories $1-3$, omitting the 14 category four cores.

\subsubsection{Temperature}

Observations of $\mathrm{CO} J=1 \rightarrow 0$ and $\mathrm{C}^{18} \mathrm{O} J=1 \rightarrow 0$ were used to estimate the kinetic temperature of each region. First, it was assumed that $\mathrm{CO} J=1 \rightarrow 0$ is optically thick and in LTE. These are reasonable assumptions given the low critical density and excitation temperature of $\mathrm{CO} J=1 \rightarrow 0$, and the large abundance of CO $J=1 \rightarrow 0$ in the ISM $\left(10^{-4}\right.$ with respect to $\mathrm{H}_{2}$ ). Under these assumptions $T_{\mathrm{mb}} \sim T_{k}$, meaning that the observed main beam temperature $\left(T_{\mathrm{mb}}\right)$ is a good approximation of the kinetic temperature of the region. $\mathrm{CO}$ and $\mathrm{C}^{18} \mathrm{O}$ emission lines were generally fit by a single Gaussian (selfabsorption effects were negligible). Next, we used our optically thin $\mathrm{C}^{18} \mathrm{O} J=1 \rightarrow 0$ as a check for these kinetic temperatures assuming an abundance ratio of $N_{\mathrm{CO}}=500 N_{\mathrm{C}^{18} \mathrm{O}}$. The results were within a few percent of the temperatures derived from the CO data, with the average kinetic temperature found to be $\sim 8 \mathrm{~K}$, ranging between $4 \mathrm{~K}$ and $16 \mathrm{~K}$. These $\mathrm{CO}$ temperatures are lower than the $15-20 \mathrm{~K}$ typically found in previous obser- 
Table 3

Observational Parameters at Center of $M S X$ Core for CS $J=5 \rightarrow 4$ and CS $J=7 \rightarrow 6$

\begin{tabular}{|c|c|c|c|c|c|c|c|c|c|c|}
\hline \multirow[b]{2}{*}{ Core } & \multirow[b]{2}{*}{$\begin{array}{l}\Delta \alpha \\
\left({ }^{\prime \prime}\right)\end{array}$} & \multirow[b]{2}{*}{$\begin{array}{l}\Delta \delta \\
\left({ }^{\prime \prime}\right)\end{array}$} & \multicolumn{4}{|c|}{$\mathrm{CS} J=5 \rightarrow 4$} & \multicolumn{4}{|c|}{$\mathrm{CS} J=7 \rightarrow 6$} \\
\hline & & & $\begin{array}{l}T_{a}^{*} \\
(\mathrm{~K}) \\
(4)\end{array}$ & $\begin{array}{c}\Delta v \\
\left(\mathrm{~km} \mathrm{~s}^{-1}\right) \\
(5)\end{array}$ & $\begin{array}{c}v_{\mathrm{lsr}} \\
\left(\mathrm{km} \mathrm{s}^{-1}\right) \\
(6)\end{array}$ & $\begin{array}{c}\int T_{a}^{*} d v \\
\left(\mathrm{~K} \mathrm{~km} \mathrm{~s}^{-1}\right) \\
(7)\end{array}$ & $\begin{array}{l}T_{a}^{*} \\
(\mathrm{~K}) \\
(8)\end{array}$ & $\begin{array}{c}\Delta v \\
\left(\mathrm{~km} \mathrm{~s}^{-1}\right) \\
(9)\end{array}$ & $\begin{array}{c}v_{\mathrm{lsr}} \\
\left(\mathrm{km} \mathrm{s}^{-1}\right) \\
(10)\end{array}$ & $\begin{array}{c}\int T_{a}^{*} d v \\
\left(\mathrm{~K} \mathrm{~km} \mathrm{~s}^{-1}\right) \\
(11)\end{array}$ \\
\hline G05.85-0.23 & 25 & -25 & $\ldots$ & $\ldots$ & $\ldots$ & $\ldots$ & $\ldots$ & $\ldots$ & $\ldots$ & $\ldots$ \\
\hline G06.26-0.51 & 0 & 0 & 0.13 & 4.07 & 19.91 & 0.56 & $\ldots$ & $\ldots$ & $\ldots$ & $\ldots$ \\
\hline G09.16+0.06 & 0 & 0 & $\ldots$ & $\ldots$ & $\ldots$ & $\ldots$ & $\ldots$ & $\ldots$ & $\ldots$ & $\ldots$ \\
\hline G09.21-0.22 & 0 & 0 & 0.08 & 2.45 & 41.73 & 0.53 & $\ldots$ & $\ldots$ & $\ldots$ & $\ldots$ \\
\hline G09.28-0.15 & 0 & 0 & 0.22 & 2.60 & 41.66 & 0.23 & $\ldots$ & $\ldots$ & $\ldots$ & $\ldots$ \\
\hline G09.86-0.04 & 0 & 0 & 0.12 & 1.50 & 17.60 & 0.19 & $\ldots$ & $\ldots$ & $\ldots$ & $\ldots$ \\
\hline G09.88-0.11 & 0 & 0 & $\ldots$ & $\ldots$ & $\ldots$ & $\ldots$ & $\ldots$ & $\ldots$ & $\ldots$ & $\ldots$ \\
\hline G10.59-0.31 & 0 & 0 & $\ldots$ & $\ldots$ & $\ldots$ & $\ldots$ & $\ldots$ & $\ldots$ & $\ldots$ & $\ldots$ \\
\hline G10.70-0.33 & 0 & 0 & $\ldots$ & $\ldots$ & $\ldots$ & $\ldots$ & $\ldots$ & $\ldots$ & $\ldots$ & $\ldots$ \\
\hline G10.99-0.09 & 0 & 0 & 0.07 & 3.20 & 30.31 & 0.08 & 0.47 & 3.20 & 29.66 & 0.83 \\
\hline G12.22+0.14 & 0 & 0 & 0.25 & 2.20 & 39.60 & 1.06 & $\ldots$ & $\ldots$ & $\ldots$ & $\ldots$ \\
\hline G12.50-0.22 & 0 & -25 & $\ldots$ & $\ldots$ & $\ldots$ & $\ldots$ & 0.46 & 2.30 & 37.27 & 2.91 \\
\hline G14.33-0.57 & 0 & 0 & $\ldots$ & $\ldots$ & $\ldots$ & $\ldots$ & $\ldots$ & $\ldots$ & $\ldots$ & $\ldots$ \\
\hline G19.28-0.39 & 0 & 0 & $\ldots$ & $\ldots$ & $\ldots$ & $\ldots$ & $\ldots$ & $\ldots$ & $\ldots$ & $\ldots$ \\
\hline G19.37-0.03 & 0 & 0 & 0.28 & 3.80 & 27.14 & 0.57 & $\ldots$ & $\ldots$ & $\ldots$ & $\ldots$ \\
\hline G19.40-0.01 & 0 & 0 & $\ldots$ & $\ldots$ & $\ldots$ & $\ldots$ & $\ldots$ & $\ldots$ & $\ldots$ & $\ldots$ \\
\hline G23.37-0.29 & 0 & 0 & $\ldots$ & $\ldots$ & $\ldots$ & $\ldots$ & $\ldots$ & $\ldots$ & $\ldots$ & $\ldots$ \\
\hline G23.48-0.53 & 0 & 0 & $\ldots$ & $\ldots$ & $\ldots$ & $\ldots$ & $\ldots$ & $\ldots$ & $\ldots$ & $\ldots$ \\
\hline $\mathrm{G} 24.05-0.22$ & 25 & 0 & $\ldots$ & $\ldots$ & $\ldots$ & $\ldots$ & 0.24 & 3.30 & 80.23 & 3.12 \\
\hline G24.16+0.08 & 0 & 0 & $\ldots$ & $\ldots$ & $\ldots$ & $\ldots$ & $\ldots$ & $\ldots$ & $\ldots$ & $\ldots$ \\
\hline G25.99-0.06 & 25 & 25 & 0.26 & 2.50 & 89.24 & 1.96 & $\ldots$ & $\ldots$ & $\ldots$ & $\ldots$ \\
\hline G30.14-0.07 & 0 & 0 & $\ldots$ & $\ldots$ & $\ldots$ & $\ldots$ & $\ldots$ & $\ldots$ & $\ldots$ & $\ldots$ \\
\hline G30.49-0.39 & 0 & 0 & $\ldots$ & $\ldots$ & $\ldots$ & $\ldots$ & $\ldots$ & $\ldots$ & $\ldots$ & $\ldots$ \\
\hline G30.53-0.27 & 0 & 0 & $\ldots$ & $\ldots$ & $\ldots$ & $\ldots$ & $\ldots$ & $\ldots$ & $\ldots$ & $\ldots$ \\
\hline G30.89+0.14 & 0 & 0 & $\ldots$ & $\ldots$ & $\ldots$ & $\ldots$ & $\ldots$ & $\cdots$ & $\cdots$ & $\cdots$ \\
\hline G30.98-0.15 & -50 & 0 & 0.26 & 3.20 & 78.02 & 1.32 & $\ldots$ & $\ldots$ & $\ldots$ & $\ldots$ \\
\hline G31.02-0.12 & -25 & 0 & $\ldots$ & $\ldots$ & $\ldots$ & $\ldots$ & $\ldots$ & $\ldots$ & $\ldots$ & $\ldots$ \\
\hline G32.01+0.05 & 0 & 0 & 0.20 & 5.40 & 95.96 & 0.46 & $\ldots$ & $\ldots$ & $\ldots$ & $\ldots$ \\
\hline G33.82-0.22 & 0 & 0 & $\ldots$ & $\ldots$ & $\ldots$ & $\ldots$ & $\ldots$ & $\ldots$ & $\ldots$ & $\ldots$ \\
\hline G34.63-1.03 & 0 & 0 & $\ldots$ & $\ldots$ & $\ldots$ & $\ldots$ & $\ldots$ & $\ldots$ & $\ldots$ & $\ldots$ \\
\hline G34.74-0.12 & -25 & 0 & $\ldots$ & $\ldots$ & $\ldots$ & $\ldots$ & $\ldots$ & $\ldots$ & $\ldots$ & $\ldots$ \\
\hline G34.78-0.80 & 0 & 0 & $\ldots$ & $\ldots$ & $\ldots$ & $\ldots$ & $\ldots$ & $\ldots$ & $\ldots$ & $\ldots$ \\
\hline G35.20-0.72 & 0 & 0 & $\ldots$ & $\ldots$ & $\ldots$ & $\ldots$ & $\ldots$ & $\ldots$ & $\ldots$ & $\ldots$ \\
\hline G37.44+0.14 & 0 & 0 & $\ldots$ & $\ldots$ & $\ldots$ & $\ldots$ & $\ldots$ & $\ldots$ & $\ldots$ & $\ldots$ \\
\hline G37.89-0.15 & 0 & -25 & $\ldots$ & $\ldots$ & $\ldots$ & $\ldots$ & $\ldots$ & $\ldots$ & $\ldots$ & $\ldots$ \\
\hline G43.64-0.82 & 0 & 0 & $\ldots$ & $\ldots$ & $\ldots$ & $\ldots$ & $\ldots$ & $\ldots$ & $\ldots$ & $\ldots$ \\
\hline G43.78+0.05 & 0 & 0 & $\ldots$ & $\ldots$ & $\ldots$ & $\ldots$ & $\cdots$ & $\ldots$ & $\ldots$ & $\ldots$ \\
\hline G50.07+0.06 & 0 & 0 & $\ldots$ & $\ldots$ & $\ldots$ & $\ldots$ & $\ldots$ & $\ldots$ & $\ldots$ & $\ldots$ \\
\hline G53.88-0.18 & 0 & 0 & $\ldots$ & $\ldots$ & $\ldots$ & $\ldots$ & $\ldots$ & $\ldots$ & $\ldots$ & $\ldots$ \\
\hline G75.75+0.75 & 0 & 0 & $\ldots$ & $\ldots$ & $\ldots$ & $\ldots$ & $\ldots$ & $\ldots$ & $\ldots$ & $\ldots$ \\
\hline G76.38+0.63 & 0 & 0 & $\ldots$ & $\ldots$ & $\ldots$ & $\ldots$ & $\ldots$ & $\ldots$ & $\ldots$ & $\ldots$ \\
\hline
\end{tabular}

vations of similar regions, often through the use of $\mathrm{NH}_{3}$ line ratios (e.g., Garay et al. 2004; Sridharan et al. 2005; Pillai et al. 2006). Such low CO temperatures could be caused if the regions are clumpy, with substructure smaller than the beam (i.e., beam filling factors $<1$ ). Therefore, given the unknown filling factor, we have assumed a canonical kinetic temperature of $15 \mathrm{~K}$ to use in all our subsequent analysis.

\subsection{2. $L V G$ Analysis}

To determine the $\mathrm{H}_{2}$ densities and CS column densities, we used a LVG code (as used by Plume et al. 1997) to solve the coupled equations of statistical equilibrium and radiative transfer. Using the assumptions of constant density and temperature with a uniformly filled beam, we created a $20 \times 20$ grid in column density per velocity interval-density space with our $\mathrm{H}_{2}$ densities ranging from $10^{4}$ to $10^{8} \mathrm{~cm}^{-3}$ and our CS column density per velocity interval ranging from $10^{11}$ to $10^{18} \mathrm{~cm}^{-2}\left(\mathrm{~km} \mathrm{~s}^{-1}\right)^{-1}$. These ranges are meant to straddle the expected values of den- sity and column density found in other high-mass star-forming regions (e.g., Plume et al. 1997). Each density-column density combination produced a series of model line intensities. These model line intensities were then compared to our CS $J=2 \rightarrow$ $1, J=3 \rightarrow 2, J=5 \rightarrow 4$, and $J=7 \rightarrow 6$ observations via a reduced $\chi^{2}$ minimization routine. Thus, the $\chi^{2}$ routine finds which of the 400 models best fit our data, providing us with the density and column density of the core.

In some sources, the higher $J$ transitions were too weak to be detected: e.g., six of categories 1-3 sources did not have detectable CS $J=5 \rightarrow 4$ nor $7 \rightarrow 6$ emission, and an additional nine sources were detected in everything but CS $J=7 \rightarrow 6$. For the sources without detectable $J=5 \rightarrow 4$ and $7 \rightarrow 6$ emission, we adopted the $1 \sigma$ rms noise level of the $J=5 \rightarrow 4$ observation to assign an upper limit to the line intensity of this transition and assumed the line width and Doppler velocity to be the same for all transitions. This provided us with at least three data points (with the third point being an upper limit in six of the sources) to 
Table 4

Physical Conditions at Central Positions

\begin{tabular}{|c|c|c|c|c|c|}
\hline $\begin{array}{l}\text { Core } \\
\text { (1) }\end{array}$ & $\begin{array}{c}\text { Category } \\
\text { (2) }\end{array}$ & $\begin{array}{c}N_{\mathrm{CO}} \\
\left(10^{18} \mathrm{~cm}^{-2}\right) \\
(3)\end{array}$ & $\begin{array}{c}N_{\mathrm{N}_{2} \mathrm{H}^{+}} \\
\left(10^{12} \mathrm{~cm}^{-2}\right) \\
(4)\end{array}$ & $\begin{array}{c}n_{\mathrm{H}_{2}} \\
\left(10^{5} \mathrm{~cm}^{-3}\right) \\
(5)\end{array}$ & $\begin{array}{c}N_{\mathrm{CS}} \\
\left(10^{12} \mathrm{~cm}^{-2}\right) \\
(6)\end{array}$ \\
\hline G05.85-0.23 & 3 & 0.77 & 1.28 & $\ldots$ & $4.55^{\mathrm{a}}$ \\
\hline G06.26-0.51 & 1 & 0.88 & $\ldots$ & 9.55 & 15.8 \\
\hline G09.16+0.06 & 1 & 0.75 & $\ldots$ & 6.03 & 3.80 \\
\hline G09.21-0.22 & 2 & $\ldots$ & 3.80 & 5.75 & 12.6 \\
\hline G09.28-0.15 & 2 & $\ldots$ & 3.90 & 12.9 & 10.0 \\
\hline G09.86-0.04 & 2 & $\ldots$ & 0.92 & 4.68 & 16.2 \\
\hline G10.99-0.09 & 2 & 1.22 & 4.46 & 6.92 & 10.0 \\
\hline $\mathrm{G} 12.22+0.14$ & 2 & $\ldots$ & 3.92 & 10.2 & 18.2 \\
\hline G12.50-0.22 & 2 & 0.86 & 3.12 & 9.33 & 10.0 \\
\hline G14.33-0.57 & 1 & 1.17 & $\ldots$ & $\ldots$ & 9.33 \\
\hline G19.37-0.03 & 2 & $\ldots$ & 3.74 & 29.5 & 44.7 \\
\hline G19.40-0.01 & $3^{\mathrm{b}}$ & $\ldots$ & 1.03 & $\ldots$ & $8.41^{\mathrm{a}}$ \\
\hline G23.37-0.29 & 2 & 2.04 & 3.06 & $\ldots$ & $21.3^{\mathrm{a}}$ \\
\hline $\mathrm{G} 23.48-0.53$ & 3 & $\ldots$ & 1.56 & $\ldots$ & $2.22^{\mathrm{a}}$ \\
\hline $\mathrm{G} 24.05-0.22$ & 2 & 1.23 & 2.97 & 63.1 & 11.0 \\
\hline $\mathrm{G} 24.16+0.08$ & 1 & 0.88 & $\ldots$ & $\ldots$ & $<1.84^{\mathrm{c}}$ \\
\hline G25.99-0.06 & 2 & $\ldots$ & 0.86 & 22.4 & 8.91 \\
\hline G30.89+0.14 & 3 & $\ldots$ & 1.34 & $\ldots$ & $3.78^{\mathrm{a}}$ \\
\hline G30.98-0.15 & 2 & $\ldots$ & 4.82 & 15.8 & 13.8 \\
\hline G31.02-0.12 & 3 & 0.67 & 1.68 & 3.24 & 6.03 \\
\hline $\mathrm{G} 32.01+0.05$ & 2 & 2.37 & 7.30 & 10.5 & 23.4 \\
\hline G34.63-1.03 & 2 & 0.31 & 1.22 & $\ldots$ & $<9.58^{\mathrm{c}}$ \\
\hline G34.74-0.12 & 2 & 1.86 & 2.04 & 4.37 & 10.7 \\
\hline G34.78-0.80 & 2 & 1.19 & 0.35 & 1.55 & 18.6 \\
\hline G35.20-0.72 & 2 & 1.09 & 3.22 & $\ldots$ & $17.6^{\mathrm{a}}$ \\
\hline G37.44+0.14 & 1 & 0.60 & $\ldots$ & 2.95 & 4.68 \\
\hline G37.89-0.15 & 2 & 0.34 & 0.48 & 1.20 & 4.79 \\
\hline
\end{tabular}

Notes. Category 4 cores have not been included in this table. A “. ..” in Columns 3 and 4 indicates no detectable emission for the given molecular line. A “..." in Column 5 indicates that LVG analysis could not be performed and therefore no density was calculated.

a Did not have enough transitions of CS for LVG analysis, CS $J=2 \rightarrow 1$ assumed to be in LTE and optically thin.

b Two cores within the field of view, central core was identified as category 3 , however the second object can be identified as category 2.

${ }^{c}$ Calculated using an upper limit to the intensity, but no detection above noise was made.

which we could fit the LVG models, since a fit to only two data points would not provide valid results. The resulting densities and column densities can be found in Table 4, Columns 5 and 6.

In an additional seven of category 1-3 sources, we detect only $J=2 \rightarrow 1$ data. In these cases, we calculated the CS column density under the assumptions that the transition was optically thin and in LTE. To verify that this assumption is acceptable, we used the full LVG model to calculate the line opacities for a simulated cloud with $N_{\mathrm{CS}}=1.21 \times 10^{13} \mathrm{~cm}^{-2}$ and $n_{\mathrm{H}_{2}}$ $=1.14 \times 10^{6} \mathrm{~cm}^{-3}$ (the typical values determined from $\mathrm{LVG}$ fitting to all cores for which we had three or four CS transitions). The resulting opacity from the model was 0.1 , which supports our optically thin assumption. A similar verification was also done for sources where the higher $J$ transitions were detected. Column densities for these sources calculated assuming that the CS $J=2 \rightarrow 1$ transition was optically thin and in LTE were within a few percent of those calculated using the full LVG analysis.

\section{DISCUSSION}

\subsection{Are These Sites of Massive Star Formation?}

To determine which cores from our selection are potential sites of massive star formation, we used a variety of observed properties as evidence, specifically line widths, and column densities, along with a comparison to previous studies of starforming regions, both low mass and high mass.

\subsubsection{Line Widths}

Crapsi et al. (2005) and Caselli et al. (2002) observed mean line widths of $\sim 0.33 \mathrm{~km} \mathrm{~s}^{-1}$ and $0.50 \mathrm{~km} \mathrm{~s}^{-1}$ for $\mathrm{N}_{2} \mathrm{H}^{+}$in low-mass star-forming regions. On the other hand, Pillai et al. (2006) and Sridharan et al. (2005) observed mean line widths of $\sim 2 \mathrm{~km} \mathrm{~s}^{-1}$ and $2.1 \mathrm{~km} \mathrm{~s}^{-1}$ respectively in regions they concluded to be in the early stages of massive star formation. In addition, Pillai et al. (2007) observed line widths of $\sim 2.7 \mathrm{~km} \mathrm{~s}^{-1}$ in potential prestellar clumps in proximity to UCHII regions. Plume et al. (1997) and Shirley et al. (2003) observed mean line widths of 4.2 and $5.6 \mathrm{~km} \mathrm{~s}^{-1}$ for CS in high-mass starforming regions. Their regions, however, are active regions of star formation suggesting that the broader line widths are caused by turbulence injected by outflows from young stars.

The observed mean CS line width in both category 2 and 3 sources is $3.0 \mathrm{~km} \mathrm{~s}^{-1}$, whereas in category 1 cores the mean CS line width is $2.5 \mathrm{~km} \mathrm{~s}^{-1}$. These are consistent with the results of Pillai et al. (2007). Based on these results, we conclude that our regions have more non-thermal support than lowmass star-forming regions, and are broad enough to be possible 


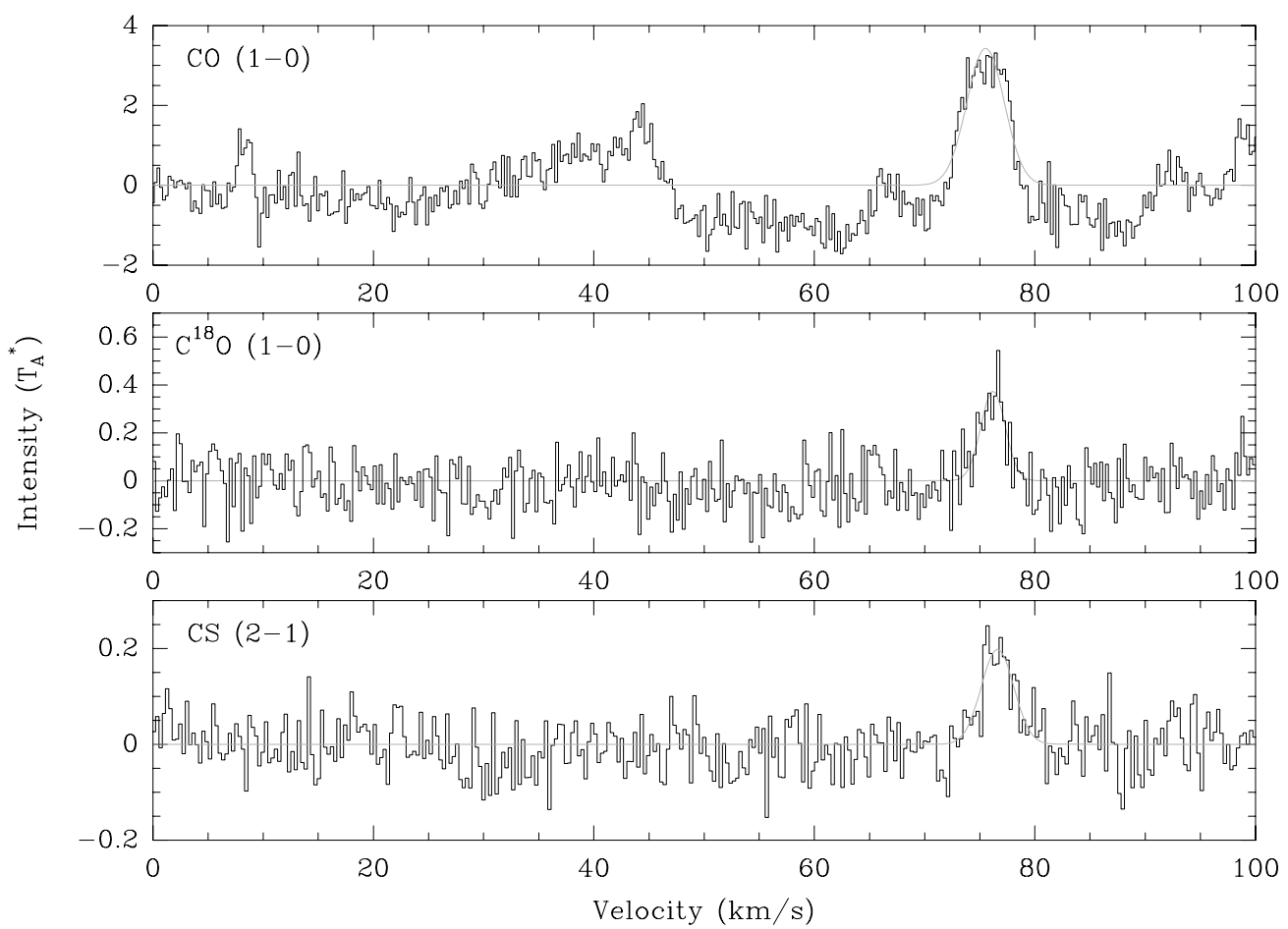

Figure 1. Determining the velocity of core G31.02-0.12. Shown are three molecules that were observed for the given core. As can be seen, though there are multiple velocity components in the CO data, the true velocity of the $M S X$ core can be determined through additional observations of another optically thin and/or high density tracer.

high-mass star-forming regions that are not currently active (see also discussion in Paper I). The fact that our lines are narrower than those found by Plume et al. (1997) and Shirley et al. (2003) suggested that our cores are more quiescent and possibly at an earlier evolutionary stage. One caveat is that line widths of $>2.0 \mathrm{~km} \mathrm{~s}^{-1}$ can occur in low-mass star-forming regions with active outflows (Rudolph et al. 2001; Tafalla et al. 2004). However, our spectra show no evidence of line wing emission which would indicate active outflows.

\subsubsection{Column Densities and Densities}

The average CS column density in Table 4 is $1.21 \times$ $10^{13} \mathrm{~cm}^{-2}$. This is approximately an order of magnitude higher than the CS column densities seen in the sample of low-mass cores by Snell et al. (1982) and Zhou et al. (1989). Our average $\mathrm{N}_{2} \mathrm{H}^{+}$column density of $7.24 \times 10^{13} \mathrm{~cm}^{-2}$ (Paper I) is also an order of magnitude higher than that found by both Caselli et al. (2002) and Crapsi et al. (2005) which were both studies of lowmass star-forming regions. See Figure 2 for the distribution of CS column densities.

Beuther et al. (2002) found $N_{\mathrm{CS}}$ to be $\sim 10^{12}$ to $10^{16} \mathrm{~cm}^{-2}$ in massive star-forming regions in the early stages of evolution. In active high-mass star-forming regions, Lada et al. (1997) found an average $N_{\mathrm{CS}}=3.98 \times 10^{13} \mathrm{~cm}^{-2}$, comparable to our results. Plume et al. (1997), however, found $N_{\mathrm{CS}}=2.63 \times 10^{14} \mathrm{~cm}^{-2}$ which is an order of magnitude higher than the CS column densities derived for our sample of cores. This is likely a real difference in column density between their maser sources and our IRDCs, and not just a modeling effect. For example, when Plume et al. (1997) modeled their same sources, but ignored the CS $J=7 \rightarrow 6$ detections, they still found $\left\langle N_{\mathrm{CS}}\right\rangle=$ $2.29 \times 10^{14} \mathrm{~cm}^{-2}$. In contrast, in their sources without any CS $J=7 \rightarrow 6$ detections, they found $N_{\mathrm{CS}}=3.71 \times 10^{13} \mathrm{~cm}^{-2}$, which is comparable to our results. Thus, the reason that we do not detect CS $J=7 \rightarrow 6$ in most of our cores is most probably a column density effect.

Figure 3 shows a histogram of the density distributions in our cores. Our average $\mathrm{H}_{2}$ density from LVG analysis is $1.1 \times$ $10^{6} \mathrm{~cm}^{-3}$. In comparison, Beuther et al. (2002) found an average $\mathrm{H}_{2}$ density of $\sim 10^{6} \mathrm{~cm}^{-3}$ in young massive star-forming regions. Plume et al. (1997) found an average of $8.5 \times 10^{5} \mathrm{~cm}^{-3}$ and Lada et al. (1997) found an average of $6.3 \times 10^{5} \mathrm{~cm}^{-3}$ in active high-mass star-forming regions. In contrast, Zhou et al. (1989) found that $2.0 \times 10^{5} \mathrm{~cm}^{-3}$ in regions forming low-mass stars. It is interesting to note that the densities of our cores are similar to those in the water maser survey of Plume et al. (1997), while our column densities are an order of magnitude lower. This may be a beam filling factor effect caused by "clumps" that are smaller than the beam. In this case, the "clumps" in our sample of IRDCs would have the same density as those in the water maser survey of Plume et al. (1997), but they would be smaller or fewer in number and, thus, fill less of the beam resulting in a lower beam-averaged column density.

Thus, the large line widths, high densities, and column densities in our sample of $M S X$ cores support the idea that these cores are, indeed, potential sites for high-mass star formation.

\subsection{Are These Objects Young?}

An understanding of interstellar chemistry combined with a model based on the object's temperature and density can provide a rough estimate to a core's age using the relative abundances of $\mathrm{C}^{18} \mathrm{O}, \mathrm{CS}$, and $\mathrm{N}_{2} \mathrm{H}^{+}$. We used a chemical evolution code developed at the University of Calgary, which, provided a series of input parameters ( $n, T, \mathrm{UV}$ field, etc.), solves the coupled differential equations which determine the abundances of each species for each time step. Thus, the code allows us to track the relative abundances of any species as a function of time. Our code incorporates gas-phase reactions, gas-grain interactions 


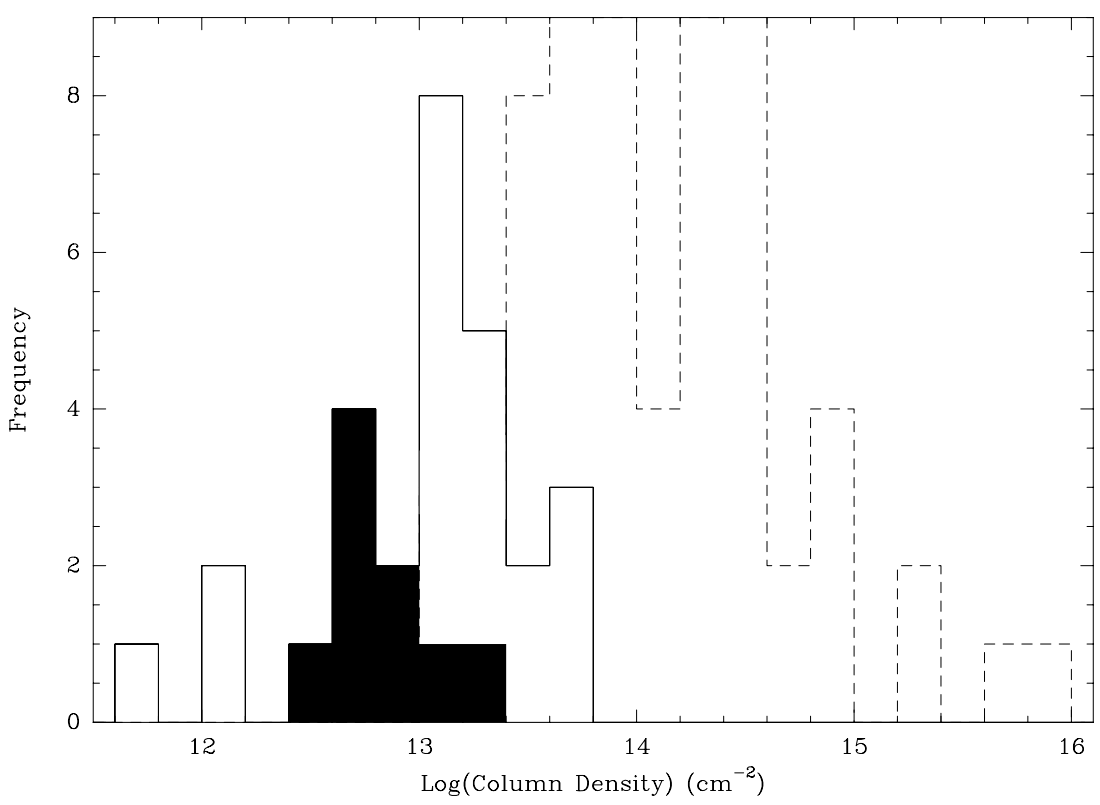

Figure 2. Solid line: histogram of the CS column densities found using LVG analysis. The average column density is $1.21 \times 10^{13} \mathrm{~cm}^{-2}$ with a $1 \sigma$ standard deviation of 0.49. Dashed line: results from Plume et al. (1997) with an average CS column density of $1.07 \times 10^{14} \mathrm{~cm}^{-2}$ (peak of histogram off scale). Filled area: results from Snell et al. (1982), with an average density of $7.53 \times 10^{12} \mathrm{~cm}^{-2}$.

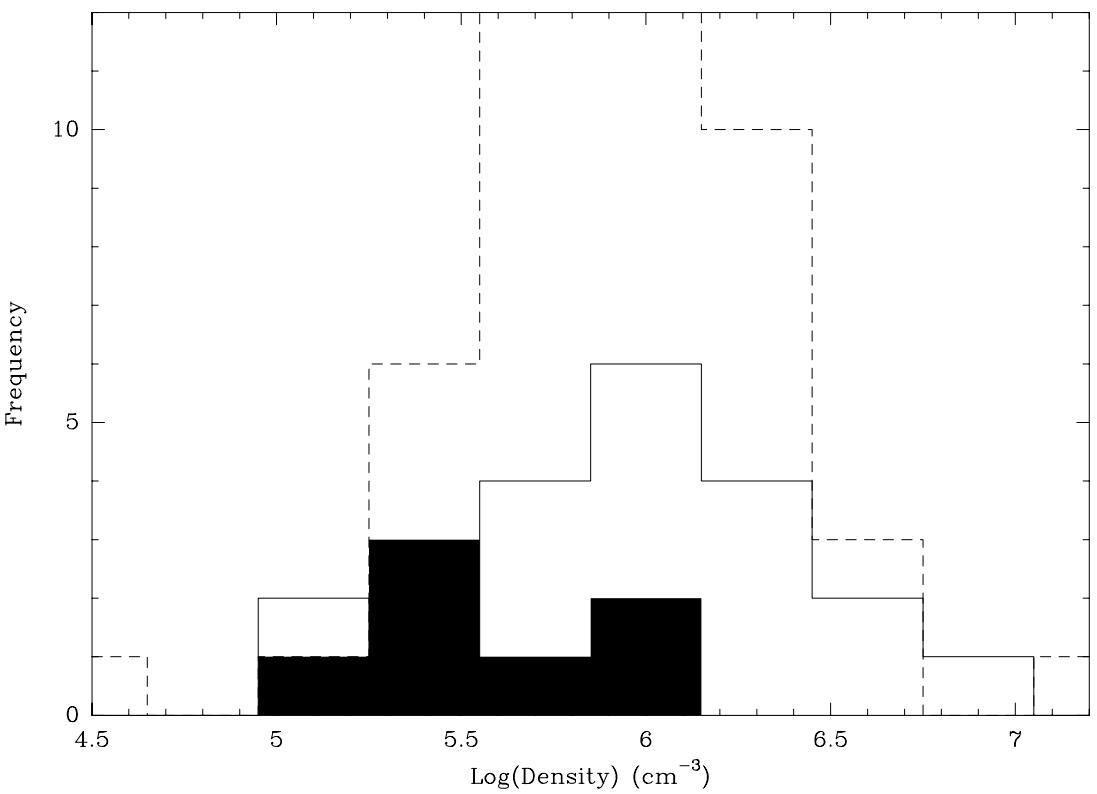

Figure 3. Solid line: histogram of the $\mathrm{H}_{2}$ densities found using LVG analysis. The average density is $1.14 \times 10^{6} \mathrm{~cm}^{-3}$ with a $1 \sigma$ standard deviation of 0.53 . Dashed line: results from Plume et al. (1997), with an average density of $8.51 \times 10^{6} \mathrm{~cm}^{-3}$ (peak of histogram off scale). Filled area: results from Lada et al. (1997), with an average density of $6.31 \times 10^{5} \mathrm{~cm}^{-3}$.

(depletion and desorption) through the treatment described by Hasegawa \& Herbst (1993), and Bergin et al. (1995), using both cosmic ray desorption and thermal evaporation as destruction mechanisms. The code utilizes the RATE99 (Le Teuff et al. 2000) reaction rate database which provides 4100 reactions connecting 400 species. Standard relative atomic abundances were used (see Table 5 which also sets the initial abundances of species at time $t=0$ ) with a set UV field strength of 1 Habing (reasonable for protostellar cores at a temperature of $15 \mathrm{~K}$ in proximity to an UCHII region; Churchwell 2002), a grain albedo of 0.6 , and an $A_{v}>100$ implying the UV field is shielded in the interior. For the sake of computational speed, we created a scaled down version of this database, with 270 species.
When tested against the full database, we saw little difference between the results.

To model our data, we ran this code using the density and temperature which emulates an average core $(n=1.14 \times$ $10^{6} \mathrm{~cm}^{-3}, T=15 \mathrm{~K}$ ). Figure 4(a) shows the results for CO, CS, and $\mathrm{N}_{2} \mathrm{H}^{+}$. It is important to note that small changes in density and temperature reflecting the range of physical conditions seen in our cores produced no significant difference in the model. In the early stages of evolution $\left(t<10^{2}\right.$ years), $\mathrm{CO}$ and CS abundances increase rapidly to their maximum level, yet there is very little (if any) $\mathrm{N}_{2} \mathrm{H}^{+}$present. This is mainly due to the difference in reaction rates and formation pathways which allow CO and CS to form rapidly. Whereas CO, as the 


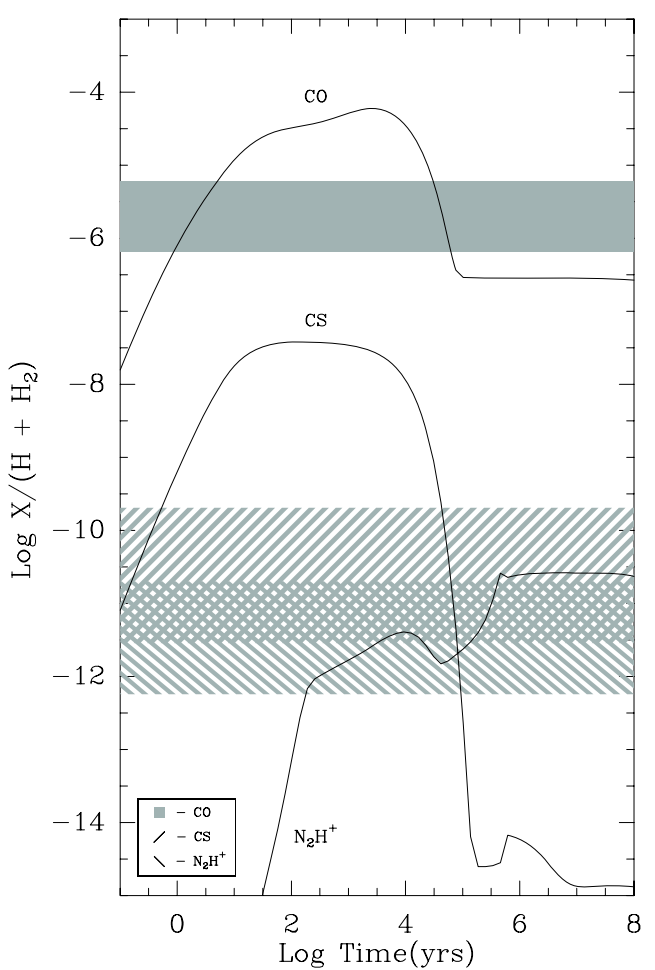

(a)

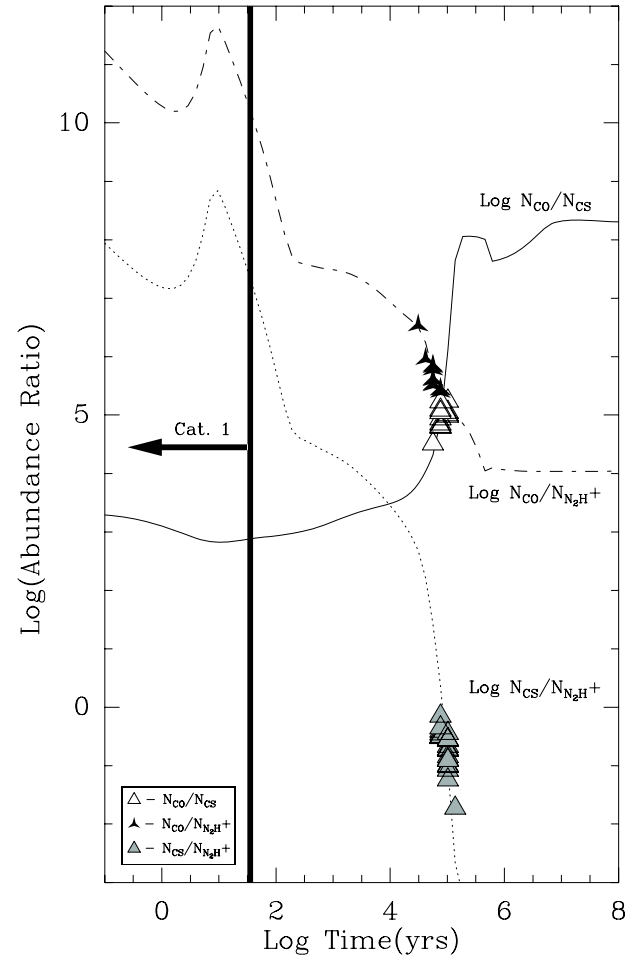

(b)

Figure 4. Standard chemical evolution model for sample cores. (a) Chemical model generated by our chemical evolution code, showing the relationship between the abundances of $\mathrm{CO}, \mathrm{CS}$, and $\mathrm{N}_{2} \mathrm{H}^{+}\left(T=15 \mathrm{~K}, n=1.14 \times 10^{6} \mathrm{~cm}^{-3}\right)$ showing chemical equilibrium being reached at $t \sim 10^{6}$ years. The range of abundances observed for each species is indicated by the listed fill pattern. (b) Abundance ratios of $N_{\mathrm{CO}} / N_{\mathrm{CS}}, N_{\mathrm{CO}} / N_{\mathrm{N}_{2} \mathrm{H}^{+}}, N_{\mathrm{CS}} / N_{\mathrm{N}_{2} \mathrm{H}^{+}}$from our model, showing an inverse relation between $\mathrm{CO}, \mathrm{CS}$, and $\mathrm{N}_{2} \mathrm{H}^{+}$. The column density ratios of category 2 and 3 cores are presented as markers on the model lines. Category 1 cores have an upper limit of $t<$ $10^{2}$ years.

(A color version of this figure is available in the online journal.)

Table 5

\begin{tabular}{lc}
\multicolumn{2}{c}{ Chemical Abundances from Model } \\
\hline \hline Species & Abundance \\
\hline$e^{-}$ & $1.46 \times 10^{-4}$ \\
$\mathrm{~N}$ & $4.50 \times 10^{-5}$ \\
$\mathrm{O}$ & $3.52 \times 10^{-4}$ \\
$\mathrm{C}^{+}$ & $1.46 \times 10^{-4}$ \\
$\mathrm{~S}^{+}$ & $4.00 \times 10^{-8}$ \\
$\mathrm{Fe}^{+}$ & $6.00 \times 10^{-9}$ \\
$\mathrm{He}^{+}$ & $2.53 \times 10^{-9}$ \\
$\mathrm{Mg}^{+}$ & $4.00 \times 10^{-9}$ \\
$\mathrm{Na}^{+}$ & $4.00 \times 10^{-9}$ \\
$\mathrm{Si}^{+}$ & $4.00 \times 10^{-8}$ \\
$\mathrm{H}_{2}$ & 1.00 \\
\hline
\end{tabular}

Note. All abundances are relative to $\mathrm{H}_{2}$.

principle destroyer of $\mathrm{N}_{2} \mathrm{H}^{+}$(Aikawa et al. 2001), hinders the increase in abundance of $\mathrm{N}_{2} \mathrm{H}^{+}$. Beyond $\sim 10^{5}$ years $\mathrm{CO}$ and $\mathrm{CS}$ begin to deplete quite readily onto dust grains which allows the $\mathrm{N}_{2} \mathrm{H}^{+}$abundance to increase. In addition, although $\mathrm{N}_{2} \mathrm{H}^{+}$can be destroyed in the gas phase through dissociative recombination with electrons, this process simply produces $\mathrm{N}_{2}$ which does not easily deplete onto dust grains but instead rapidly reacts with $\mathrm{H}_{3}{ }^{+}$to reform $\mathrm{N}_{2} \mathrm{H}^{+}$(Nakano \& Umebayashi 1986).

Although Figure 4(a) presents molecular abundance relative to the total hydrogen abundance $\left(\mathrm{H}+\mathrm{H}_{2}\right)$, we do not have direct information on the hydrogen abundance in these cores for us to directly compare our observed abundances to the model. However, by using the ratios of $N_{\mathrm{CO}} / N_{\mathrm{CS}}, N_{\mathrm{CO}} / N_{\mathrm{N}_{2} \mathrm{H}^{+}}$, and
$N_{\mathrm{CS}} / N_{\mathrm{N}_{2} \mathrm{H}^{+}}$, we eliminate the $\mathrm{H}$ abundance allowing us to compare our observations to the model and assign a relative chemical age. Nevertheless in Figure 4(a), we attempt to provide absolute abundances based on approximate $\mathrm{H}_{2}$ column densities from Paper I by convolving our MSX images to the resolution of our FCRAO data and using the simple relation $\tau_{\lambda}=\sigma_{\lambda}$. $N\left(\mathrm{H}_{2}\right)$, where $\tau_{\lambda}$ is the dust opacity, $\sigma_{\lambda}$ is the dust extinction cross section $\left(2.3 \times 10^{-23} \mathrm{~cm}^{2}\right.$ at $8.8 \mu \mathrm{m}$; Indebetouw et al. $2005)$, and $N\left(\mathrm{H}_{2}\right)$ is the column density of molecular hydrogen.

Figure 4(b) shows the column density ratio plots on which we have placed the positions of each core. The average CS column density and relative abundance of category 1 cores are $7.1 \times 10^{12} \mathrm{~cm}^{-2}$ and $1.22 \times 10^{-10}$, respectively, however with no detectable $\mathrm{N}_{2} \mathrm{H}^{+}$abundance above our detection limits $(X /(\mathrm{H}$ $\left.+\mathrm{H}_{2}\right) \sim 2.0 \times 10^{-13}$ ) these objects must be chemically young $\left(t<10^{2}\right.$ years). An alternate interpretation of these data is that there is an embedded source in the vicinity which could release $\mathrm{CO}$ and CS from grain mantles and destroy $\mathrm{N}_{2} \mathrm{H}^{+}$(e.g., Lee et al. 2004). In this instance, the core would be more evolved but would appear to be chemically younger since the chemical clock has been reset. Careful analysis of the Spitzer images by Ragan et al. (2009), however, reveals no $24 \mu \mathrm{m}$ sources coincident with category 1 sources G06.26-0.51 and G06.16+0.06 indicating that they may, indeed, be extremely young objects devoid of any star formation activity (unless any existing $24 \mu \mathrm{m}$ sources are extremely deeply embedded). G37.44+0.14, however, appears to contain a Class I protostar and, our cursory inspection of the Spitzer data for G24.16+0.08, also indicates the presence of $24 \mu \mathrm{m}$ source coincident with the $8 \mu \mathrm{m}$ dark image (there are no Multiband Imaging Photometer for Spitzer (MIPS) data for 
G14.33-0.57). Thus, it is possible that these objects are older but have had their chemical clocks reset due to heating by the embedded sources.

Category 2 cores, which have a detection of all three molecular tracers, have an apparent ambiguity in their relative ages as their CO and CS abundances can indicate an extremely chemically young ( $t<10$ years) or more chemically evolved core $\left(t>10^{4.5}\right.$ years). However, the absolute abundance of $\mathrm{N}_{2} \mathrm{H}^{+}$at the younger ages would be well below our detection limit and inspection of Figure 4(b) shows that the abundance ratios also preclude the possibility of these young ages. Thus, we conclude that these cores are indeed more chemically evolved. In category 3 cores, we see reduced abundances of CS $\left(X /\left(\mathrm{H}+\mathrm{H}_{2}\right) \sim\right.$ $\left.1.04 \times 10^{-10}\right)($ compared to the average relative $\mathrm{CS}$ abundance from our full sample) coupled with a larger column density of $\mathrm{N}_{2} \mathrm{H}^{+}\left(X /\left(\mathrm{H}+\mathrm{H}_{2}\right) \sim 3.43 \times 10^{-11}\right)$, which could be evidence of depletion. In low-mass star-forming regions depletion is dominant in the time period before protostellar formation (indicative of a more chemically evolved region), and a similar trend is expected to be seen for high-mass star-forming regions. Looking at the observed column density ratios versus those from our model (see Figure 4(b)), we assign an age range of $10^{4.6}<t$ $<10^{5.1}$ years for category 3 objects. This suggests that these sources are more chemically evolved and could be at the onset of star formation.

It is evident from Figure 4(b) that, despite our different definitions, category 2 and 3 objects appear to have similar chemical ages. The presence of an embedded star would certainly allow $\mathrm{CO}$ to evaporate off dust grains giving it the appearance of a chemically younger object (category 2) despite being more chemically evolved (category 3 ). However, the similarity in ages is more likely a result of the steepness of the abundances profiles in log space. Around $10^{5}$ years, large differences in CS or CO column densities translate into fairly small changes in time. Since we have limited observational sensitivity, the corresponding chemical ages of category 2 and 3 cores will necessarily be similar.

The absolute ages of the cores, however, are also subject to model assumptions, such as our depletion and desorption rates. Bergin et al. (1995) found that desorption of species from grain mantles has a strong dependence on its surface binding energy, which is highly uncertain and depends upon which species is the dominant component of the grain mantle. We tested this effect on our results by adjusting our binding energies and cosmic ray desorption flux by as little as $10 \%$, which resulted in the chemical ages changing by as much as a factor of a few. Therefore given our observational errors and uncertainties in our modeling, we cannot provide an absolute ages for category 2 and 3 cores but suggest that they are somewhere between $10^{4.5}<t<10^{5.5}$ years. We also cannot provide a firm age for category 1 cores, but their complete lack of $\mathrm{N}_{2} \mathrm{H}^{+}$emission is a strong argument in favor of their extreme youth.

\subsection{A Diversity of IRDCs}

In Paper I, we presented core masses based upon observations of $\mathrm{C}^{18} \mathrm{O} J=1 \rightarrow 0$ and $\mathrm{N}_{2} \mathrm{H}^{+} J=1 \rightarrow 0$, with kinematic distances calculated using the line center velocity and the Milky Way rotation curve model of Fich et al. (1989). However, in the early stages of core evolution mass can be primarily accounted for by $\mathrm{C}^{18} \mathrm{O}$, whereas in later stages when it is depleted, the sum of this depleted $\mathrm{C}^{18} \mathrm{O}$ and the newly formed $\mathrm{N}_{2} \mathrm{H}^{+}$is likely a better representation of the overall mass. Therefore, in Table 6, Column 6 of this paper, we provide the sum of these masses.
As a means of comparison to these molecular line based core masses, it is useful to calculate the virial mass of the cores. We used the relation:

$$
M_{\text {Virial }}=\frac{5}{3} \frac{R V_{\mathrm{rms}}^{2}}{G},
$$

where $R$ is the radius of the cloud, $G$ is the gravitational constant, and $V_{\text {rms }}=3^{\frac{1}{2}} \Delta V / 2.35$, where $\Delta V$ is the average FWHM line width of the CS observations. Since the virial mass is only dependent on the radius (and not the cube of the radius) it is much less sensitive to errors in the assumed distance to the cloud. Virial masses of the cores can be found in Table 6, Column 5.

Comparison of the molecular line based masses to the virial masses may provide information about the structure and state of the cores. The average $M_{\text {Virial }} / M_{\text {Molecular }}$ is 0.78 suggesting that the clouds are roughly in virial equilibrium. Note, however, that in only four cases $M_{\text {Virial }}>M_{\text {mol }}$ whereas, in the rest, $M_{\text {Virial }}<M_{\text {mol }}$. These discrepancies could be caused by a number of factors. For example, if the clouds are clumpy with beam filling factors less than 1 , the observed intensity will be lower than it would be for a uniformly filled beam, resulting in lower column density values and, therefore, artificially lowered values of $M_{\mathrm{mol}}$. Indeed in a sub-sample of our cores, Ragan et al. (2009) find clear evidence for significant sub-structure on scales below the beam size of all our molecular observations. Other possibilities are the existence of streaming motions adding to the line width of the observations resulting in artificially increased values of $M_{v i r}$. Alternately, the cores may not actually be in virial equilibrium, suggesting that they are not gravitationally bound and may, in fact, be transient objects (Ballesteros-Paredes 2006). Finally, our acceptance of standard abundance ratios may be naïve, as many studies have shown this value to vary with location (e.g., Wall 2007).

Comparison of the Jeans mass to the mass calculated from our molecular observations allows us to estimate if our cores are gravitationally unstable and whether or not the core has the potential to form a single star or a cluster. Using a canonical temperature of $15 \mathrm{~K}$ and the density results from the LVG model, we used the following reduced form of the Jeans mass relation:

$$
M_{\mathrm{Jeans}}=\left(\frac{5 k T}{G \mu \mathrm{m}_{H}}\right)^{\frac{3}{2}}\left(\frac{3}{4 \pi \rho_{0}}\right)^{\frac{1}{2}} M_{\odot},
$$

where $\rho_{0}$ is the density of the region and $\mu$ is the mean mass per particle (2.2). Jeans masses of the cores can be found in Table 5, Column 4. Cores with only one CS transition observed or detected do not have a Jeans mass since, in these cases, LVG modeling to obtain their densities could not be undertaken.

Table 6 shows that almost all sources we observed have masses substantially larger than the Jeans mass, in many cases 2-3 orders of magnitude higher in value, suggesting that these sources will not form individual protostars but rather protoclusters. This is not unusual since star formation is known to be strongly clustered (Lada et al. 1997; Bonnell \& Davies 1998) and IRDCs are clearly the precursors to stellar clusters (e.g., Rathborne et al. 2006, Ragan et al. 2009).

Ragan et al. (2009) performed a detailed investigation of the association of mid-IR absorbing cloud with young stars as identified by the Spitzer bands in a sub-sample of sources in this paper. For all sources they found that most of the cloud mass is unassociated with deeply embedded young protostars. However, all of the cores in their smaller Spitzer sub-sample have YSOs present in the near vicinity, just beyond the confines 
Table 6

Table of Masses

\begin{tabular}{|c|c|c|c|c|c|}
\hline $\begin{array}{l}\text { Core } \\
\text { (1) }\end{array}$ & $\begin{array}{c}\text { Distance } \\
(\mathrm{kpc}) \\
(2)\end{array}$ & $\begin{array}{c}\text { Radius } \\
\text { (pc) } \\
(3)\end{array}$ & $\begin{array}{c}M_{\text {Jeans }} \\
\left(M_{\odot}\right) \\
(4)\end{array}$ & $\begin{array}{c}M_{\text {Virial }} \\
\left(10^{3} M_{\odot}\right) \\
(5)\end{array}$ & $\begin{array}{c}M_{\text {Molecular }} \\
\left(10^{3} M_{\odot}\right) \\
(6)\end{array}$ \\
\hline G05.85-0.23 & 3.14 & 0.38 & $\ldots$ & 0.39 & 0.51 \\
\hline G06.26-0.51 & 3.78 & 0.92 & 6 & 3.46 & 6.20 \\
\hline G09.16+0.06 & 3.81 & 1.73 & 7 & 0.76 & 3.30 \\
\hline G09.21-0.22 & 4.57 & 0.83 & 8 & 1.12 & 1.40 \\
\hline G09.28-0.15 & 4.48 & 1.09 & 5 & 1.64 & 3.40 \\
\hline G09.86-0.04 & 2.36 & 0.79 & 8 & 0.68 & 1.50 \\
\hline G10.99-0.09 & 3.32 & 1.51 & 7 & 3.48 & 6.30 \\
\hline $\mathrm{G} 12.22+0.14$ & 3.75 & 0.68 & 6 & 0.70 & 0.32 \\
\hline G12.50-0.22 & 3.55 & 1.08 & 6 & 1.20 & 7.64 \\
\hline G14.33-0.57 & 2.05 & 0.31 & 7 & 0.36 & 0.83 \\
\hline G19.37-0.03 & 2.26 & 0.27 & 3 & 0.85 & 0.28 \\
\hline G19.40-0.01 & 2.23 & 0.27 & $\ldots$ & 0.53 & 2.10 \\
\hline G23.37-0.29 & 4.70 & 1.42 & $\ldots$ & 6.52 & 7.40 \\
\hline $\mathrm{G} 23.48-0.53$ & 4.10 & 0.50 & $\ldots$ & 1.84 & 2.70 \\
\hline $\mathrm{G} 24.05-0.22$ & 4.82 & 1.31 & 2 & 2.34 & 2.50 \\
\hline $\mathrm{G} 24.16+0.08$ & 3.46 & 0.42 & $\ldots$ & $\ldots$ & 2.60 \\
\hline G25.99-0.06 & 5.15 & 0.78 & 4 & 1.04 & 0.68 \\
\hline G30.89+0.14 & 5.65 & 2.05 & $\ldots$ & 0.24 & 11.00 \\
\hline G30.98-0.15 & 4.63 & 0.56 & 5 & 1.28 & 1.90 \\
\hline G31.02-0.12 & 4.56 & 1.11 & 10 & 2.54 & 7.30 \\
\hline $\mathrm{G} 32.01+0.05$ & 5.77 & 0.52 & 6 & 3.24 & 21.70 \\
\hline G34.63-1.03 & 0.84 & 0.10 & $\ldots$ & $\ldots$ & 0.12 \\
\hline G34.74-0.12 & 4.86 & 0.74 & 9 & 1.88 & 3.27 \\
\hline G34.78-0.80 & 2.80 & 1.19 & 15 & 2.73 & 2.90 \\
\hline G35.20-0.72 & 2.17 & 0.26 & $\ldots$ & 0.58 & 2.50 \\
\hline G37.44+0.14 & 2.60 & 0.39 & 11 & 0.18 & 0.09 \\
\hline G37.89-0.15 & 0.82 & 0.30 & 17 & 0.04 & 0.06 \\
\hline
\end{tabular}

Notes. "..." indicates that LVG analysis could not be performed. Therefore, an $\mathrm{H}_{2}$ density was not available to calculate the Jeans mass.

of the mid-IR dark cloud. Thus, to the limit of the Spitzer observations, which is to below a solar mass at the cloud surface, and larger masses for deeply embedded sources, the bulk of the molecular material appears pre-stellar. In particular, G05.85-0.23 and G37.89-0.15 had no embedded sources at $24 \mu \mathrm{m}$ within the boundaries of the cores, with a maximum of three embedded sources found along the edges of G34.63-1.03 and G37.44-0.14. Labeled a category 1 object, the embedded sources within G37.44-0.14 likely evaporated $\mathrm{CO}$ from the dust grains and destroyed $\mathrm{N}_{2} \mathrm{H}^{+}$, which would cause an error in our chemical model interpretation.

In this regard, there are two cores that stand out as somewhat different from the rest of the sample: G37.44-0.14 and G37.89-0.15. These cores appear to have molecular masses sufficient for the formation of massive stars. (Sridharan et al. (2005) found masses to be a few $\times 10^{2}$ to $10^{3} M_{\odot}$ for high-mass preprotostellar objects.) In addition, their virial and jeans masses suggest that these objects are likely gravitational bound and massive enough to collapse. However unlike other sources that also pass these criteria, their cloud masses are only 8 and 3 times larger than their Jeans masses and their densities are about an order of magnitude below the average value. Thus, these cores will likely form less massive clusters or possibly even single, massive stars. Although, in the latter case, this single "isolated" massive star would likely be forming in the presence of other YSOs in the context of a larger cluster. For example, in G37.44, there are other $8 \mu \mathrm{m}$ dark regions in the $M S X$ image presented in Paper I which may all be related to a larger complex. These results suggest a diversity in the type of stellar systems that can be formed within infrared dark clouds-from very massive clouds that will create large clusters, to smaller clouds that are analogs of some of the local star-forming regions like Serpens and Ophiuchus.

\section{CONCLUSIONS}

We have identified 27 possible protostellar objects through the observation of molecular tracers $\mathrm{C}^{18} \mathrm{O}, \mathrm{CS}$, and $\mathrm{N}_{2} \mathrm{H}^{+}$. By calculating their column densities and densities, we determined their evolutionary states based on a comparison to a chemical evolution model. In Paper I, the primary focus was on the morphologies of the molecular emission of these tracers, with rough estimates for their sizes and densities. In this paper, we used the following evidence to identify these sources as potential sites of massive star formation:

1. Our average CS column density $\left(1.21 \times 10^{13} \mathrm{~cm}^{-2}\right)$ and $\mathrm{H}_{2}$ density $\left(1.14 \times 10^{6} \mathrm{~cm}^{-3}\right)$ is higher then those found in low-mass star-forming regions (e.g., Snell et al. 1982) and comparable to those found in high-mass star-forming regions (e.g., Plume et al. 1997).

2. Our average CS line widths $\left(\Delta v \sim 2.0-3.0 \mathrm{~km} \mathrm{~s}^{-1}\right)$ are comparable to high-mass star-forming regions (e.g., Pillai et al. 2007), and larger than low-mass star-forming regions (e.g., Crapsi et al. 2005).

3. Our core masses are larger than their Jeans masses suggesting they are of sufficient mass to collapse. In addition, $M_{\text {Virial }} / M_{\text {Molecular }}=0.78$ which suggests that these regions are likely gravitationally bound. 
4. By comparing our molecular abundances to a chemical model, we found our sources to be chemically young $\left(10^{4.5}<t<10^{5.5}\right.$ years $)$ for categories 2 and $3, t<10^{2}$ years for category 1 ) suggesting that these regions may not have yet formed massive protostars.

In addition, we have explored the generic star-forming properties within the sample and find some diversity in the types of stellar systems that can be formed within IRDCs thereby extending the range of infrared dark clouds from the very massive clouds that will create large clusters to clouds that are analogs of some of the local star-forming regions. Multiwavelength studies of the internal structure of these cores using the Spitzer Space Telescope (Ragan et al. 2009) along with high-resolution molecular observations to further constrain their temperature and density structure will help to provide a more accurate picture of these regions as well as the nature of their embedded stellar populations.

The authors would like to thank the anonymous referee who provided many useful comments and who helped to significantly improve this paper.

\section{REFERENCES}

Aikawa, Y., Ohashi, N., Inutsuka, S., Herbst, E., \& Takakuwa, S. 2001, ApJ, 552, 639

Ballesteros-Paredes, J. 2006, MNRAS, 372, 443

Bergin, E. A., Langer, W. D., \& Goldsmith, P. F. 1995, ApJ, 441, 222

Bergin, E. A., \& Tafalla, M. 2007, ARA\&A, 45, 339

Beuther, H., Schilke, P., Menten, K. M., Motte, F., Sridharan, T. K., \& Wyrowski, F. 2002, ApJ, 566, 945

Beuther, H., \& Sridharan, T. K. 2007, ApJ, 668, 348

Beuther, H., \& Steinacker, J. 2007, ApJ, 656, L85

Beuther, H., Walsh, A., Schilke, P., Sridharan, T. K., Menten, K. M., \& Wyrowski, F. 2002, A\&A, 390, 289

Bonnell, I. A., \& Davies, M. B. 1998, ApJ, 295, 691

Butler, M. J., \& Tan, J. C. 2009, ApJ, 696, 484

Carey, S. J., Clark, F. O., Egan, M. P., Price, S. D., Shipman, R. F., \& Kuckar, T. A. 1998, ApJ, 508, 721

Caselli, P., Benson, P. J., Myers, P. C., \& Tafalla, M. 2002, ApJ, 572, 238

Chambers, E. T., Jackson, J. M., Rathborne, J. M., \& Simon, R. 2009, ApJS, 181,360

Churchwell, E. 2002, ARA\&A, 40, 27

Crapsi, A., Caselli, P., Walmsley, C. M., Myers, P. C., Tafalla, M., Lee, C. W., \& Bourke, T. L. 2005, ApJ, 619, 379

Dopita, M. 1991, PASA, 9, 234

Du, F., \& Yang, J. 2008, ApJ, 686, 384

Fich, M., Blitz, L., \& Stark, A. 1989, ApJ, 342, 272

Garay, G., Faúndez, S., Mardones, D., Bronfman, L., Chini, R., \& Nyman, L. 2004, ApJ, 610, 313

Garay, G., \& Lizano, S. 1999, PASP, 111, 1049

Hasegawa, T. I., \& Herbst, E. 1993, MNRAS, 261, 83

Indebetouw, R., et al. 2005, ApJ, 619, 931
Knez, C., Shirley, Y. L., Evans, N. J. II, \& Mueller, K. E. 2002, in ASP Conf Ser. 267, Hot Star Workshop III: The Earliest Stages of Massive Star Birth, ed. P. A. Crowther (San Francisco, CA: ASP), 375

Kutner, M. L., \& Ulich, B. L. 1981, ApJ, 250, 341

Lada, C. J., \& Lada, E. A. 2003, ARA\&A, 41, 57

Lada, E. A., Strom, K. M., \& Myers, P. C. 1993, in Protostars and Planets III, ed. E. H. Levy \& J. I. Lunine (Tucson: Univ. Arizona Press), 245

Lada, E. A., Evans, N. J. II, \& Falgarone, E. 1997, ApJ, 488, 286

Lee, J.E., Bergin, E. A., \& Evans, N. J. II 2004, ApJ, 617, 360

Le Teuff, Y. H., Millar, T. J., \& Markwick, A. J. 2000, A\&A, 146, 157

Leurini, S., Schilke, P., Wyrowski, F., \& Menten, K. M. 2007, A\&A, 466, 215

McKee, C. F. 1986, Ap\&SS, 118, 383

McKee, C. F., \& Tan, J. C. 2003, ApJ, 585, 850

Menten, K. M., Pillai, T., \& Wyrowski, F. 2005, in IAU Symp. Proc. Int. Astronomical Union 227, Massive Star Birth: A Crossroads of Astrophysics, ed. R. Cesaroni, M. Felli, E. Churchwell, \& M. Walmsley (Cambridge: Cambridge Univ. Press), 23

Molinari, S., Brand, J., Cesaroni, R., \& Palla, F. 1996, A\&A, 308, 573

Mueller, K. E., Shirley, Y. L., Evans, N. J. II, \& Jacobson, H. R. 2002, ApJS, 143,469

Nakano, T., \& Umebayashi, T. 1986, MNRAS, 221, 319

Pillai, T., Wyrowski, F., Carey, S. J., \& Menten, K. M. 2006, A\&A, 450, 569

Pillai, T., Wyrowski, F., Hatchell, J., Gibb, A. G., \& Thompson, M. A. 2007, A\&A, 467, 207

Plume, R., Jaffe, D. T., \& Evans, N. J., II. 1992, ApJS, 78, 505

Plume, R., Jaffe, D. T., Evans, N. J. II, Martin-Pintado, J., \& Gomez-Gonzalez, J. 1997, ApJ, 476, 730

Price, S. D., et al. 1996, BAAS, 28, 1341

Ragan, S.E., Bergin, E.A., \& Gutermuth, R.A. 2009, ApJ, 698, 324

Ragan, S. E., Bergin, E. A., Plume, R., Gibson, D. L., Wilner, D. J., O’Brien, S., \& Hails, E. 2006, ApJS, 166, 567

Rathborne, J. M., Jackson, J. M., \& Simon, R. 2006, ApJ, 641, 389

Rathborne, J. M., Jackson, J. M., Zhang, Q., \& Simon, R. 2008, ApJ, 689, 1141

Redman, R. O., Feldman, P. A., Côté, S., Wyrowski, F., Carey, S. J., \& Egan, M. P. 2002, in ASP Conf. Ser. 267, Hot Star Workshop III: The Earliest Stages of Massive Star Birth, ed. P. A. Crowther (San Francisco, CA: ASP), 409

Rudolph, A. L., Bachiller, R., Rieu, N. Q., Van Trung, D., Palmer, P., \& Welch, W. J. 2001, ApJ, 558, 204

Shirley, Y. L., Evans, N. J. II, Young, K. E., Knez, C., \& Jaffe, D. T. 2003, ApJS, 149,375

Snell, R. L., Langer, W. D., \& Frerking, M. A. 1982, ApJ, 255, 149

Sridharan, T. K., Beuther, H., Saito, M., Wyrowski, F., \& Shilke, P. 2005, ApJ, 634,57

Sridharan, T. K., Beuther, H., Shilke, P., Menten, K., \& Wyrowski, F. 2002, ApJ, 566, 931

Tafalla, M., Myers, P. C., Caselli, P., \& Walmsley, C. M. 2004, Ap\&SS, 292, 347

Wall, W. F. 2007, MNRAS, 379, 674

Wang, Y., Zhang, Q., Rathborne, J. M., Jackson, J., \& Wu, Y. 2006, ApJ, 651, L125

Wu, Y., Zhang, Q., Chen, H., Yang, C., \& Ho, P. T. P. 2000, in ASP Conf. Ser. 217, Imaging at Radio through Submillimeter Wavelengths, ed. J. G. Mangum \& S. J. E. Radford (San Francisco, CA: ASP), 98

Zhou, S., Wu, Y., Evans, N. J. II, Fuller, G. A., \& Myers, P. C. 1989, ApJ, 346, 168 